\title{
Allosteric Antagonist Modulation of TRPV2 by Piperlongumine Impairs Glioblastoma Progression
}

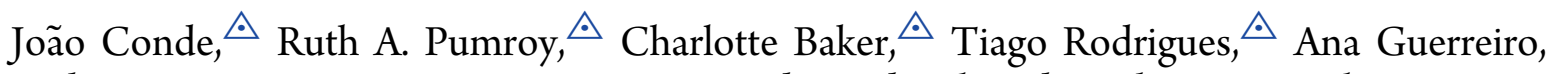
Bárbara B. Sousa, Marta C. Marques, Bernardo P. de Almeida, Sohyon Lee, Elvira P. Leites, Daniel Picard, Amrita Samanta, Sandra H. Vaz, Florian Sieglitz, Maike Langini, Marc Remke, Rafael Roque, Tobias Weiss, Michael Weller, Yuhang Liu, Seungil Han, Francisco Corzana, Vanessa A. Morais, Cláudia C. Faria, Tânia Carvalho, Panagis Filippakopoulos, Berend Snijder, Nuno L. Barbosa-Morais, Vera Y. Moiseenkova-Bell,* and Gonçalo J. L. Bernardes*

Cite This: ACS Cent. Sci. 2021, 7, 868-881

Read Online

ACCESS |

山ll Metrics \& More

回国 Article Recommendations

Supporting Information

ABSTRACT: The use of computational tools to identify biological targets of natural products with anticancer properties and unknown modes of action is gaining momentum. We employed self-organizing maps to deconvolute the phenotypic effects of piperlongumine (PL) and establish a link to modulation of the human transient receptor potential vanilloid 2 (hTRPV2) channel. The structure of the PL-bound full-length rat TRPV2 channel was determined by cryo-EM. PL binds to a transient allosteric pocket responsible for a new mode of anticancer activity against glioblastoma (GBM) in which hTRPV2 is overexpressed. Calcium

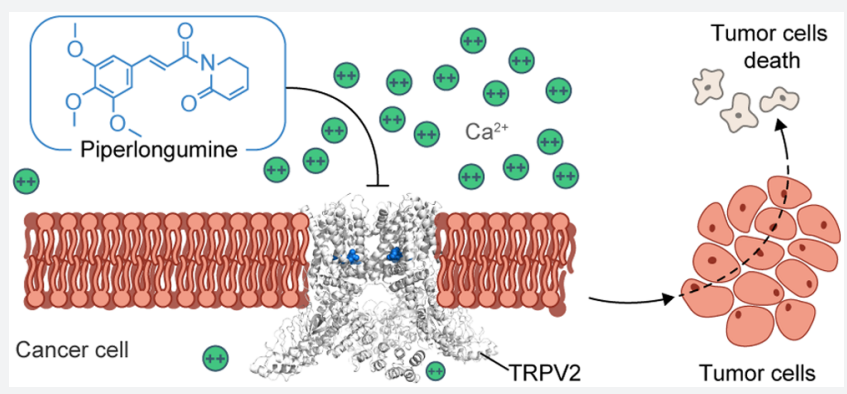
imaging experiments revealed the importance of Arg539 and

Thr522 residues on the antagonistic effect of PL and calcium influx modulation of the TRPV2 channel. Downregulation of hTRPV2 reduces sensitivity to PL and decreases ROS production. Analysis of GBM patient samples associates hTRPV2 overexpression with tumor grade, disease progression, and poor prognosis. Extensive tumor abrogation and long term survival was achieved in two murine models of orthotopic GBM by formulating PL in an implantable scaffold/hydrogel for sustained local therapy. Furthermore, in primary tumor samples derived from GBM patients, we observed a selective reduction of malignant cells in response to PL ex vivo. Our results establish a broadly applicable strategy, leveraging data-motivated research hypotheses for the discovery of novel means tackling cancer.

\section{INTRODUCTION}

Natural products provide ample opportunities to develop innovative medicines. ${ }^{1-4}$ However, understanding their mechanisms of action remains a bottleneck to unlock their promise in drug discovery. ${ }^{5-9}$ Chemoproteomics is a privileged approach to unveil new biology for molecules of therapeutic interest. However, such methods are laborious and timeconsuming and unlikely identify membrane proteins and targets with only minute expression. ${ }^{9}$ Moreover, chemoproteomics relies on structural manipulation of the chemical entities under study, which may have unpredictable outcomes. $^{10}$ Considering the large volume of publicly available bioactivity data, statistical learning of ligand-target relationships has become tractable in recent years and amenable to providing probabilistically motivated target binding hypotheses. ${ }^{7,11}$ One such ligand-based target-identification program (SPiDER: self-organizing map-based prediction of drug equivalence relationships $)^{11}$ has been validated for the deorphanization of natural products and discovery of new biology, ${ }^{7,12}$ drug repurposing, ${ }^{13,14}$ and the unravelling of primary and secondary pharmacology. ${ }^{2}$ Indeed, in silico technologies are a growing concept in drug discovery that may offer complementary/alternative solutions for not only target identification programs ${ }^{7,14}$ but also discovery chemistry in general. $^{15}$

Piperlongumine (PL) is an alkaloid that has attracted much attention as a selective anticancer agent despite its unclear pharmacology and mode of action. ${ }^{16-18}$ Thus, unveiling the underlying pharmacology can open new research avenues and leverage the development of $\mathrm{PL}$ as an efficacious cancer treatment. Here, we used SPiDER, as an alternative to

Received: January 15, 2021

Published: April 14, 2021

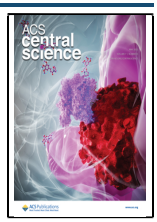




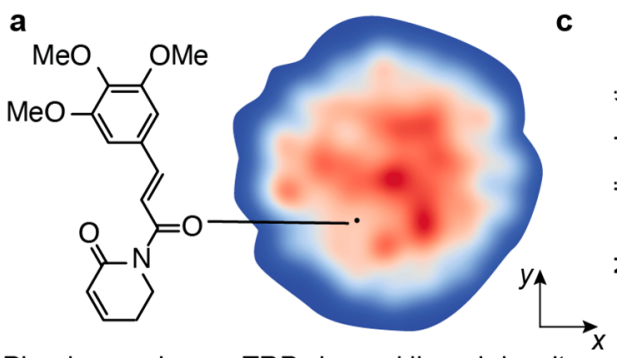

Piperlongumine TRP channel ligand density

\begin{tabular}{ll}
$\begin{array}{l}\text { b } \\
\text { TRP } \\
\text { channel }\end{array}$ & IC $_{50} / \boldsymbol{\mu M} \pm \mathbf{S D}$ \\
\hline TRPV1 & $>100$ \\
TRPV2 & $4.6 \pm 0.13$ log units \\
TRPV3 & $>100$ \\
TRPV4 & $>100$ \\
TRPV5 & $>100$ \\
TRPA1 & $39 \pm 0.15$ log units \\
TRPC1 & $>100$ \\
TRPC3 & $>100$ \\
TRPC4 & $>100$ \\
TRPC5 & $>100$ \\
TRPC6 & $>100$ \\
TRPC7 & $64 \pm 0.06$ log units \\
TRPM2 & $>100$ \\
TRPM3 & $>100$ \\
TRPM5 & $>100$ \\
TRPM8 & $>100$ \\
\hline TRP
\end{tabular}

SD $=$ standard deviation

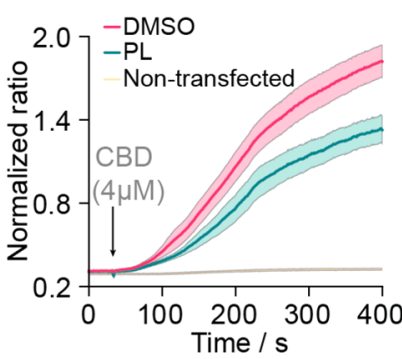

d

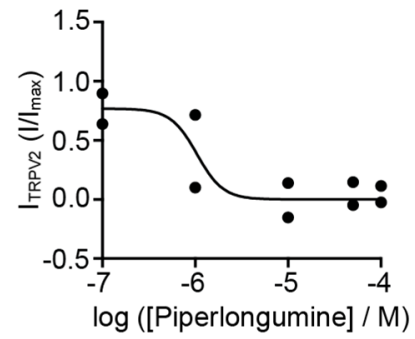

f
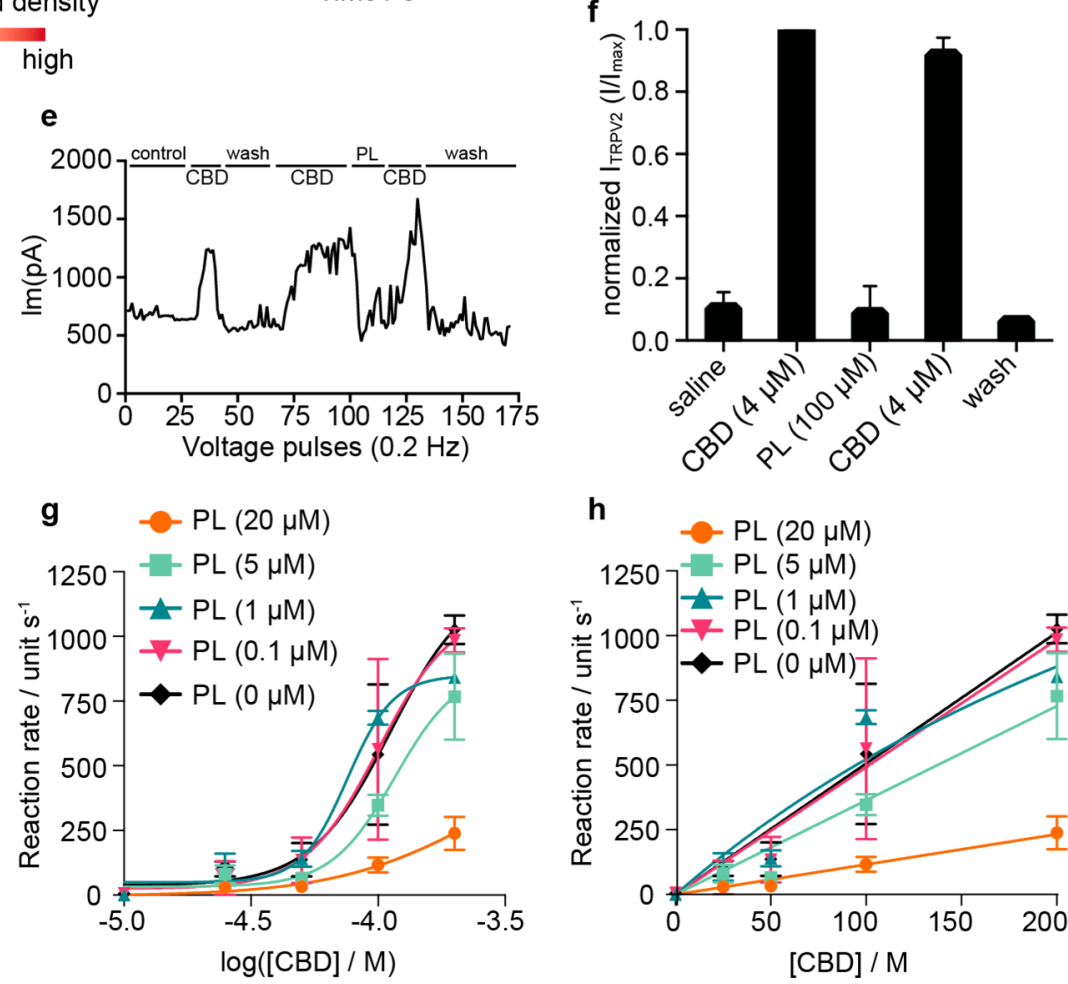

h
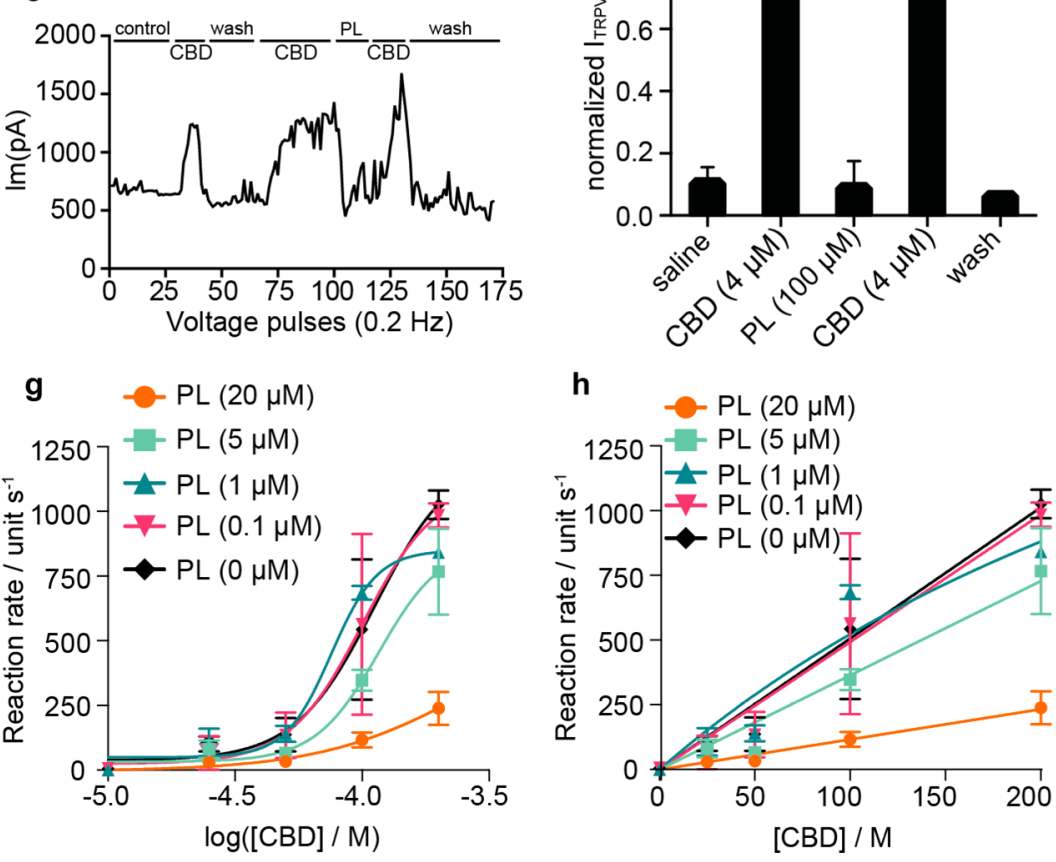

Figure 1. SPiDER identifies piperlongumine as a selective, reversible, and allosteric antagonist of human TRPV2 (hTRPV2). (a) Manifold learning ( $t$-distributed stochastic neighborhood embedding) of transient receptor potential (TRP) channel ligand data as described by CATS2 descriptors. PL (black dot) presents an uncommon pharmacophore among known TRP ligands as it is located in a region of low ligand density. (b) PL is a selective hTRPV2 antagonist. Controls-hTRPV2, tranilast $\left(\mathrm{IC}_{50}=2.3 \mu \mathrm{M} \pm 0.25 \log\right.$ units); hTRPA1, ruthenium red $\left(\mathrm{IC}_{50}=2.3 \mu \mathrm{M} \pm 0.19 \log \right.$ units); hTRPC7, SKF 96365 ( IC $_{50}=103 \mu \mathrm{M} \pm 0.27 \log$ units); $n=2$. (c) Inhibition of cannabidiol (CBD, $\left.4 \mu \mathrm{M}\right)$ evoked calcium influx by PL (5 $\mu \mathrm{M})$ in hTRPV2-transfected HEK293T cells. For quantification, only the strongest responding cells were analyzed $(n=30-40$ cells). Shaded regions correspond to the $95 \%$ confidence interval of the mean response. $* * * * p<0.0001$ (DMSO versus PL; DMSO versus nontransfected; oneway ANOVA, Dunnett post hoc test) at 300 s poststimulation. (d) Concentration-response curve of PL against hTRPV2 with a patch-clamp assay. $\mathrm{IC}_{50}=1 \mu \mathrm{M} \pm 0.52$ log units, $n=2$, stimulus: $4 \mu \mathrm{M}$ CBD. (e) Exemplary patch-clamp time-course trace that shows the inhibitory effect of PL (100 $\mu \mathrm{M})$ against the CBD-activated $(4 \mu \mathrm{M})$ hTRPV2 currents; $n=2$. Data are consistent with reversible binding. (f) Normalized responses of the drug wash-out experiment. Data are consistent with reversible binding. $(\mathrm{g}) \mathrm{EC}_{50}$ plots for CBD with increasing concentrations of PL. (h) MichaelisMenten plots of $\mathrm{CBD} / \mathrm{PL}$ coincubation. Decreasing $\mathrm{EC}_{50}$ and $v_{\max }$ values are consistent with allosteric inhibition by PL.

chemical proteomics, to identify potential biological targets for PL that could be pursued in downstream validation studies. We report on an unprecedented link between PL and the human transient receptor potential vanilloid 2 (hTRPV2) channel. To achieve this, we performed extensive experimental validation-including cryoelectron microscopy (cryo-EM)to illuminate the PL-TRPV2 molecular recognition mechanism. Our studies reveal a new, transient binding site in TRPV2, whose recognition results in selective, potent allosteric antagonism by PL. Moreover, we found that the anticancer activity of PL is high in cell lines with high hTRPV2 expression, which is a marker of poor prognosis in glioblastoma multiforme (GBM). Most importantly, local (intracranial) and sustained delivery of PL in mice with GBM using a bespoke material produced a potent and selective antitumor effect and almost complete remission of the disease. Additionally, we observed a selective reduction of malignant cells in response to PL ex vivo in primary tumor samples derived from GBM patients. These results open up new vistas for successful GBM therapies.

\section{RESULTS AND DISCUSSION}

Self-Organizing Maps Identify TRP Channels as Targets of Piperlongumine. We used the SPiDER method $^{7,13}$ that employs self-organizing maps and topological descriptors to infer biological targets for small molecules. By correlating PL to the structure of reference ligands, targets were predicted (Table S1). We prioritized TRP channels for screening given the high prediction confidence and availability of assays for panel screening. Moreover, a privileged link 


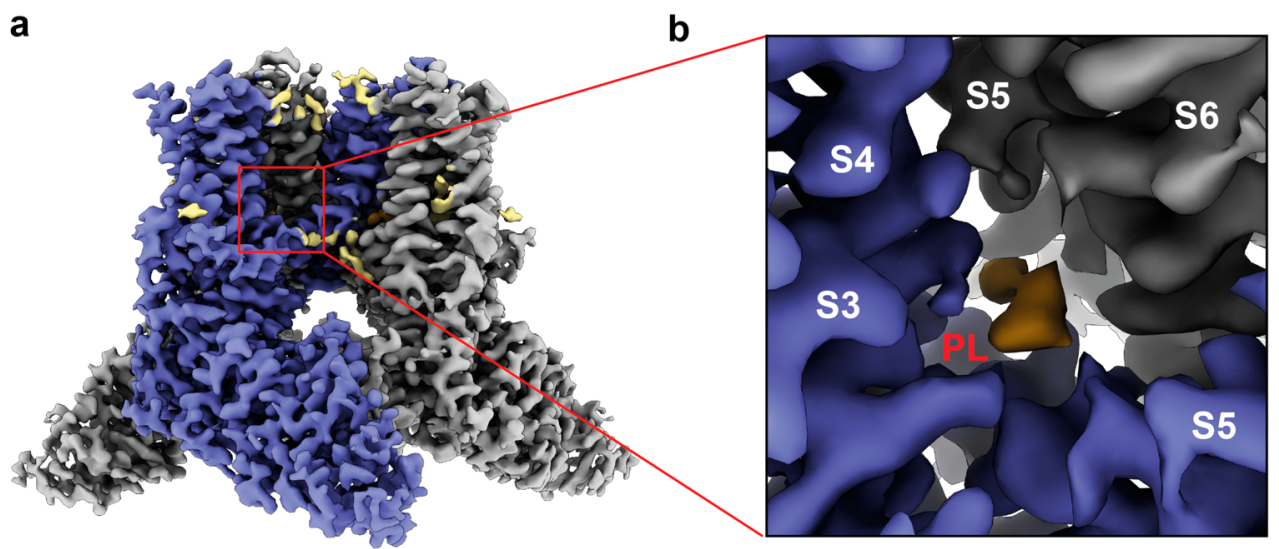

C
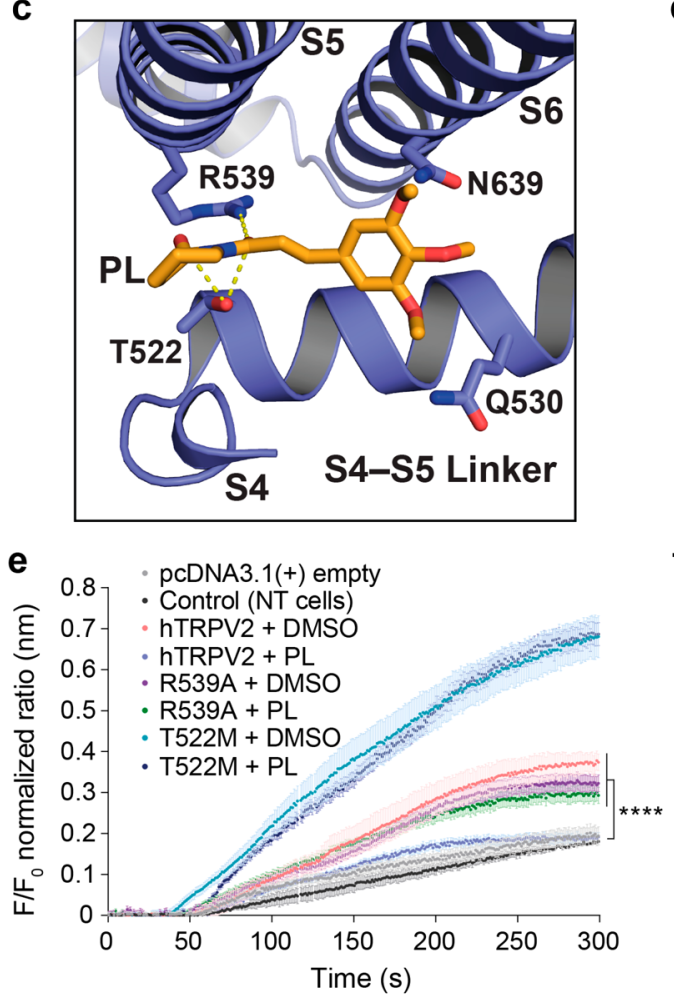

d

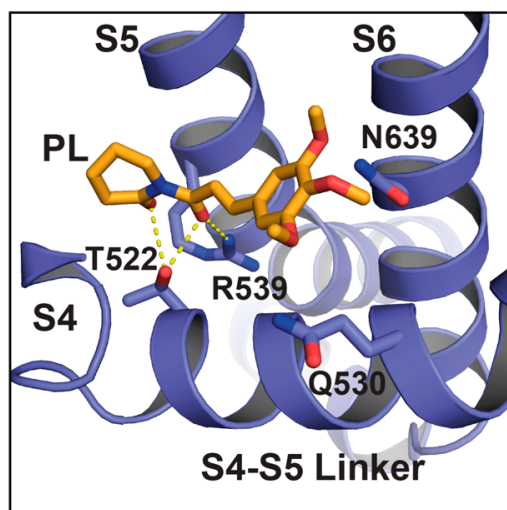

$\mathbf{f}$

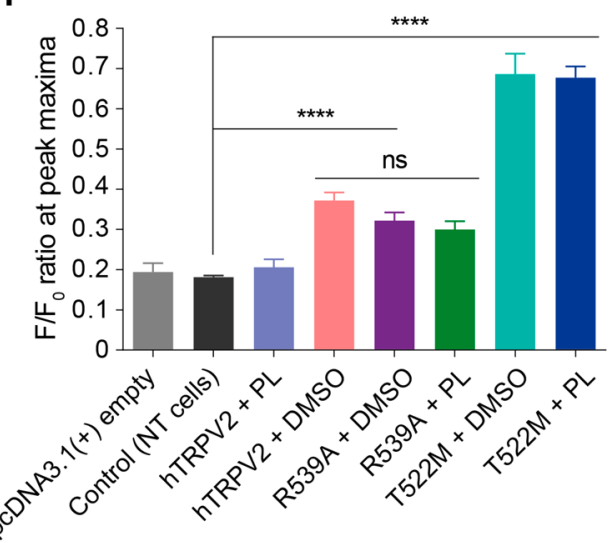

Figure 2. PL binding pocket revealed by cryo-EM. Cryo-EM map of PL-bound TRPV2 (a) and a close up of the PL-binding site (b). A single monomer is colored purple while the others are gray; lipids are colored khaki, and density for PL is colored orange. (c, d) Cartoon and stick representation of the PL binding site from two different angles, with TRPV2 represented in purple and PL in orange. Effect of PL (5 $\mu \mathrm{M})$ on cannabidiol (CBD, $4 \mu \mathrm{M}$ ) induced calcium influx in HEK293T cells, transfected with hTRPV2-RFP and variants (R539A and T522M). (e) Inhibitory potential of piperlongumine in cells transfected with hTRPV2-RFP and variants (R539A and T522M), measured by calcium imaging. For quantification, 40-60 responding cells were analyzed in each well $(n=3)$, and values were normalized to the baseline. One-way ANOVA of significant difference between the control (NT cells) and other represented groups: $p<0.0001(* * * *), p>0.05$ (n.s.). Values represent mean \pm SEM. (f) Bar graph of peaks at $260 \mathrm{~s}$ poststimulation. Two-way ANOVA for multiple comparisons between groups: $p<0.0001(* * * *), p>0.05$ (n.s.).

between several members of the TRP channel family and natural products had been established. ${ }^{2,8,19,20}$ We further motivated screening of PL against TRP channels by processing the SPiDER output with a data visualization algorithm. PL was projected onto a region of low TRP ligand density, which suggests that its pharmacophore has been scarcely employed to interrogate TRP-channel biology as opposed to molecules within other regions of chemical space (Figure 1a).
To validate the drug-target prediction, we tested PL in functional, cell-based fluorescence assays. PL showed antagonism of hTRPV2 $\left(\mathrm{IC}_{50}=4.6 \mu \mathrm{M} \pm 0.13 \mathrm{log}\right.$ units $)$ and selectivity against a TRP channel panel (Figure 1b). Agonistic activity was not detected in any case. In calcium-imaging experiments with HEK293T cells transiently transfected with hTRPV2-RFP, we confirmed significant inhibition $(p<0.0001$; one-way ANOVA and Dunnett post hoc test) of calcium influx evoked by cannabidiol-an hTRPV2 agonist (Figure 1c). 
a

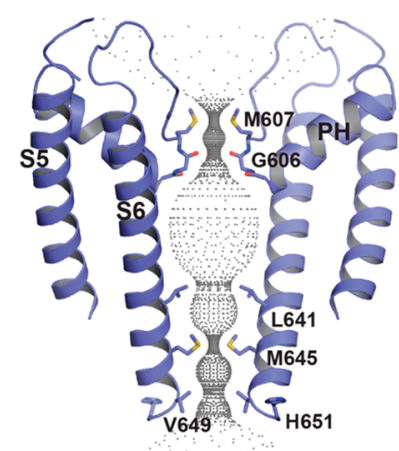

C

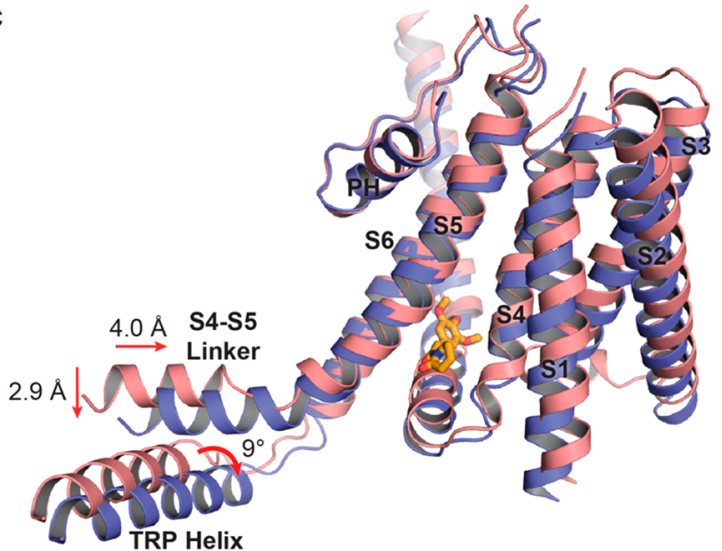

b

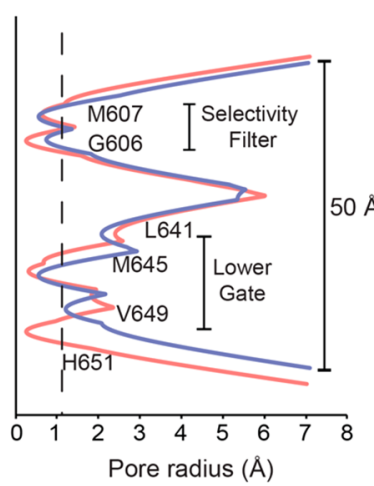

d

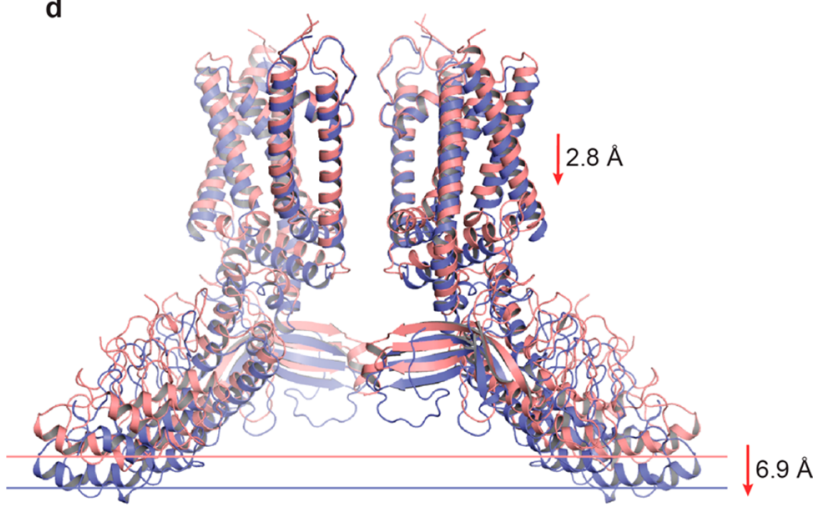

Figure 3. Conformational changes to TRPV2 on PL binding. (a) Ion permeation pathway of PL-bound TRPV2. Two opposing monomers are depicted as cartoons; gray dots represent open volume along the ion permeation pathway. (b) Pore profile of PL-bound TRPV2 (purple) and apo TRPV2 (PDB 6U84) (pink). The radius of a dehydrated calcium ion (1.1 $\AA$ ) is depicted as a dashed black line. Cartoon representations of PLbound TRPV2 (purple) and apo TRPV2 (PDV 6U84) showing shifts around the S4-S5 linker (c) and overall structural changes (d).

Similarly, PL inhibited calcium influx evoked by cannabidiol $\left(\mathrm{IC}_{50}=1 \mu \mathrm{M} \pm 0.52 \log\right.$ units; Figure 1d, Figure S1) in a patch-clamp assay. The Michael acceptor motifs in PL have been previously implicated in covalent and irreversible modification of proteins. ${ }^{18}$ To confirm that PL reversibly inhibits hTRPV2, we performed wash-out assays. The functional hTRPV2 activity could be reinstated, which shows that binding is either noncovalent or that PL presents a fast offrate after covalent modification of hTRPV2 (Figure 1e,f). This observation contrasts with a recent report on the covalent and stable modification of GSTO1 by PL. ${ }^{21}$ We next synthesized two PL analogues, as a racemic mixture, comprising a cyclopropane ring and without Michael acceptor motifs. The trans derivative displayed a perfect alignment with that of PL (Figure S2). No functional activity was found for both small molecules (Figure S3), suggesting a potential role of the conjugated motifs for molecular recognition, namely, for inducing a bioactive conformation. To further understand modulation of TRPV2 by PL, we probed whether PL and cannabidiol compete for the same binding pocket through a series of coincubation experiments. The maximal effect and apparent $v_{\max }$ value of cannabidiol decreased with increasing PL concentrations (Figure 1g,h). Both observations are consistent with noncompetitive (allosteric) antagonism. ${ }^{22}$ Neither autofluorescence nor SCAM behavior ${ }^{23}$ that could interfere with assay readouts was detected at relevant assay concentrations (Table S2).

Molecular Mechanism of Piperlongumine Interaction with TRPV2. We used cryo-EM to gain atomistic insight into the molecular mechanism of TRPV2 antagonism by PL. We reconstituted the full-length rat TRPV2 (rTRPV2, 77\% identity and 90\% similarity to hTRPV2) into nanodiscs and incubated with $50 \mu \mathrm{M} \mathrm{PL}\left(\approx 10\right.$ times the $\left.\mathrm{EC}_{50}\right)$ before preparing grids for cryo-EM. This yielded an rTRPV2-PL complex data set with a single state at $3.5 \AA$ with C4 symmetry (Table S3 and Figures S4 and S5). This state was similar to the apo truncated rabbit TRPV2 in amphipols (PDB 5AN8) and the cannabidiol (CBD) state 2 rTRPV2 in nanodisc (PDB 6U88) (Figure 2a, Figures S4 and S5) structures determined previously. $^{24-27}$ The reconstructed map has the highest quality in the transmembrane region, but no density is resolved for the pore turret (residues Ser564-Pro587).

Recent structures of rTRPV2 in nanodiscs show density for lipid tails in the vanilloid pocket; ${ }^{25}$ these densities are absent in the PL-bound cryo-EM map (Figure S6). Instead, we observed an elongated density starting at the vanilloid pocket and stretching along the top of the S4-S5 linker that we have attributed to PL. This density is wedged above the S4-S5 linker in a pocket formed by the S4 helix and the S5 and S6 helices of an adjacent TRPV2 monomer (Figure 2b, Figure S6). The trimethoxylphenyl moiety in PL is oriented toward the C-terminal end of the S4-S5 linker and the vanilloid pocket, whereas the dihydropyridinone group is oriented toward the $\mathrm{N}$-terminal end (Figure $2 \mathrm{c}$ ). $\mathrm{PL}$ is stabilized in this new allosteric pocket by hydrogen bridges between the two carbonyl groups of PL and the side-chains of Thr522 and Arg539 (Figure 2d). A density similar to that of PL is not 

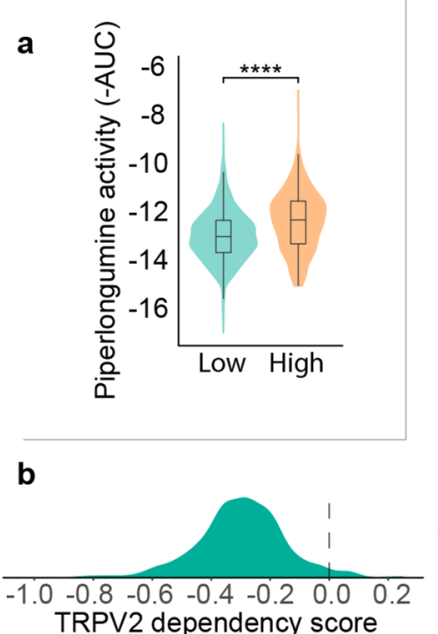

TRPV2 dependency score

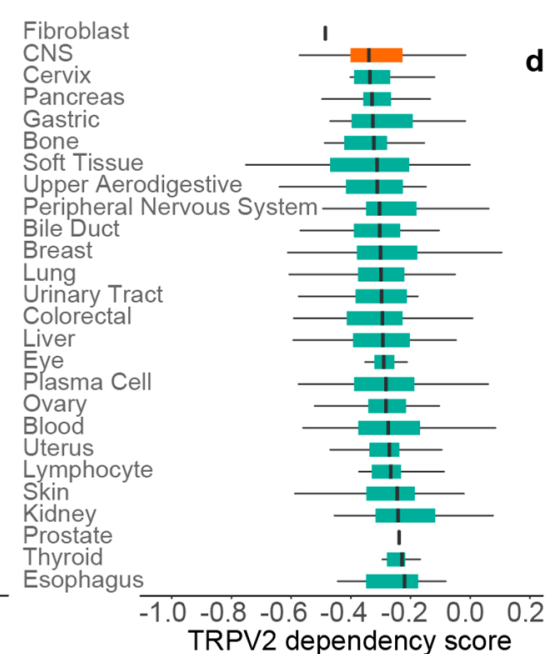

d

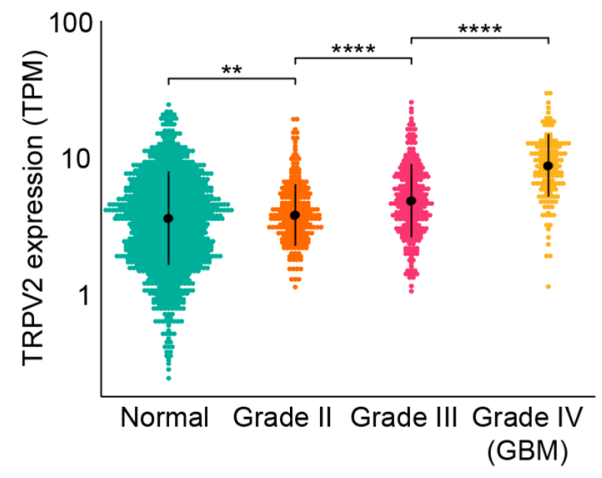

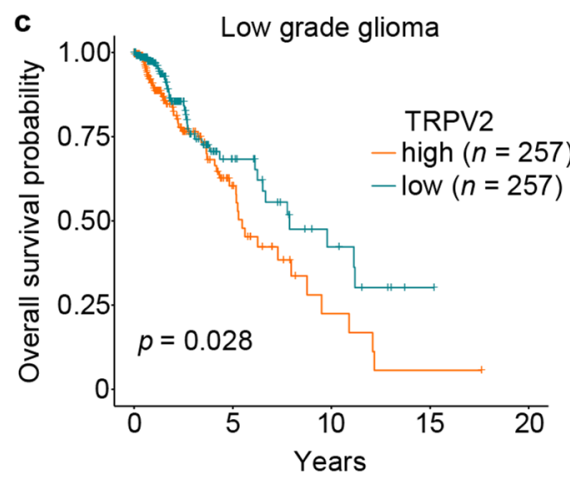

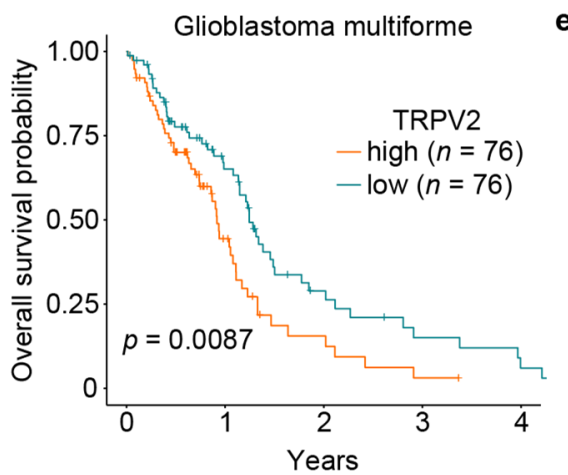

e GBM
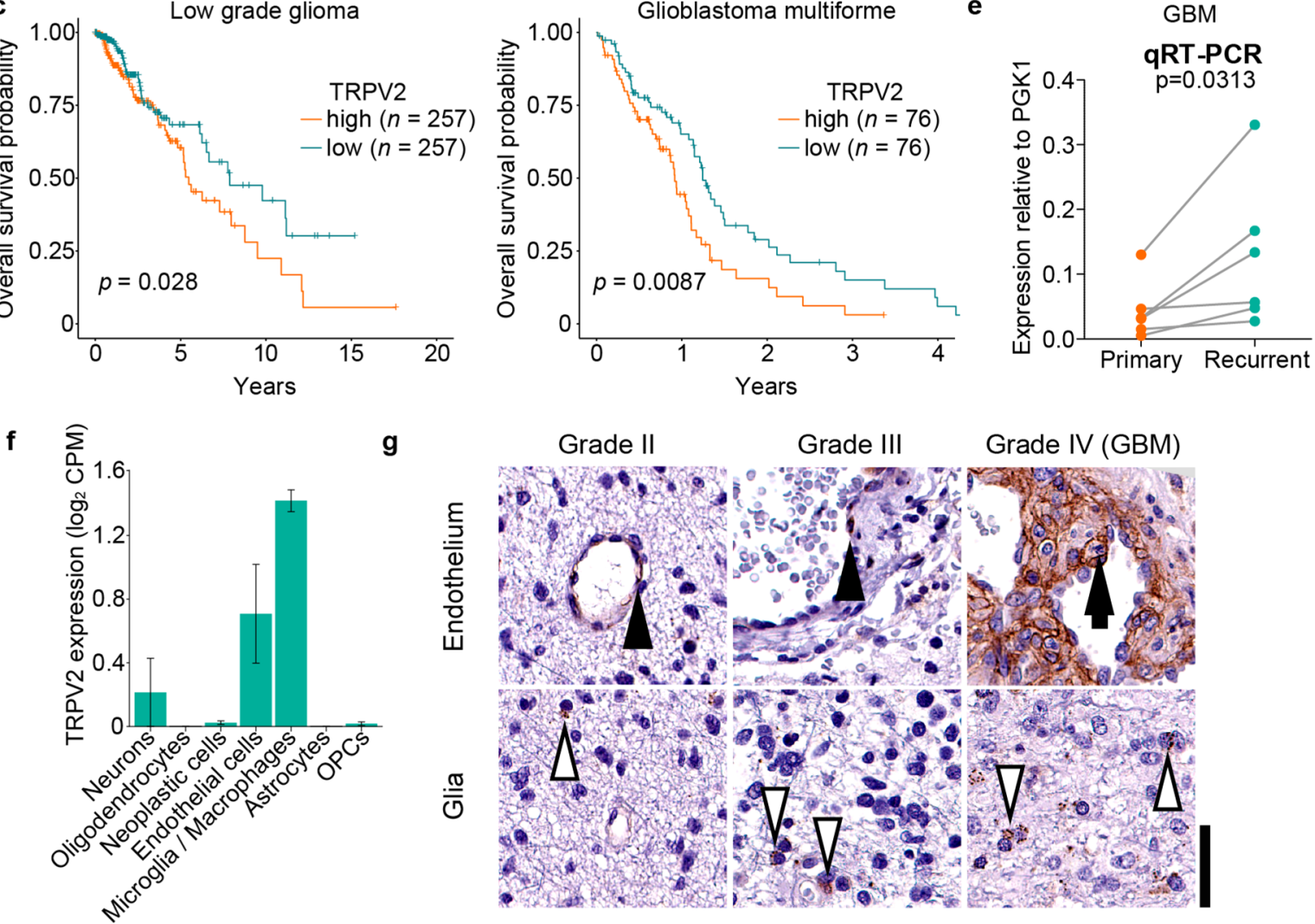

g

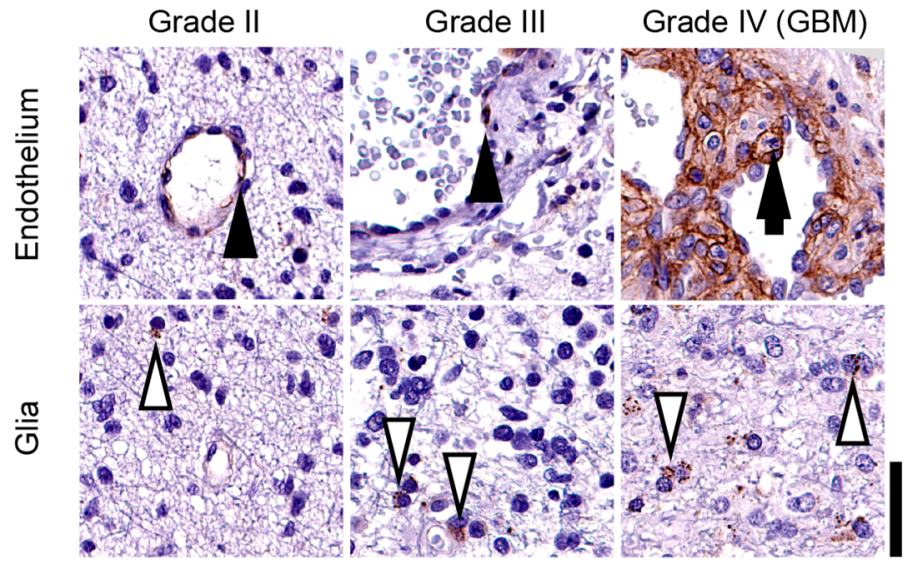

Figure 4. hTRPV2 expression is required by CNS cancer cell lines and associated with disease progression and poor prognosis in glioblastoma multiforme. (a) Cell lines with high hTRPV2 expression (as defined in Figure S12a) are sensitive to PL activity $(-$ AUC). **** represents $p<$ 0.0001 (Wilcoxon rank-sum test). (b) Cell lines from the Central Nervous System (CNS) lineage are the most sensitive to hTRPV2 loss. The effect of hTRPV2 loss-of-function was quantified with the CERES dependency score: a lower score represents a higher likelihood that the gene is essential in a given cell line. Left: smoothed histogram of distribution of hTRPV2 dependency scores across all cell lines. (c) hTRPV2 expression is a marker for poor prognosis in human gliomas. Overall survival Kaplan-Meier plots for patient stratification based on hTRPV2 median expression in lowand high-grade gliomas. $p$ values for log-rank tests for differences in survival are shown. (d) hTRPV2 expression increases concomitantly with brain tumor stage. hTRPV2 expression across GTEx normal and TCGA tumor (grouped in LGG grade II, LGG grade III, and GBM) brain samples. Black points and lines represent the median \pm upper/lower quartiles. ** represents $p<0.01$ and $* * * * p<0.0001$ (Wilcoxon rank sum test). (e) qRT-PCR of hTRPV2 in matched primary and recurrent GBM pairs. PGK1 was used as an internal control, and the Wilcoxon matched pairs signed rank test was used for statistics. (f) hTRPV2 is expressed in endothelial and microglia/macrophage brain tumor cells. hTRPV2 expression (log 2 of counts per million, CPM) across 3589 single cells derived from four GBM human samples, grouped by cell type. Data are shown as mean \pm standard error. (g) hTRPV2 is expressed in diffuse human gliomas, in glial (white arrowhead) and in endothelial cells (black arrowhead); and endothelial cells in GBM display an intense and diffuse staining of the cytoplasmic membrane in areas of microvascular proliferation, which contrasts with the weak or negative staining of the endothelial cells of grade II and III gliomas, and with the cytoplasmic and punctate staining pattern of glial cells. 
present in any currently published TRPV2 X-ray or cryo-EM map $^{24-28}$ (Figure S6).

rTRPV2 Arg539 (Arg537 in humans) is conserved as either Arg or Lys across the TRPV family in humans. Thus, while it may be involved in $\mathrm{PL}$ recognition, it is unlikely to account for TRPV2 selectivity. Moreover, Thr522 (Thr520 in humans) is highly conserved across TRPV2 in different species but is not conserved across the TRPV family in humans (Figure S7). While the Thr520/522 position is also a Thr in human TRPV4, it is a Met in TRPV3 and the otherwise highly homologous TRPV1 (Figure S7). Altogether, this may make Thr520/522 key for PL specificity. To challenge this hypothesis, validate the binding site, and test the contribution of these two residues to the effect of PL, we mutated Arg539 to Ala and Thr522 to Met in hTRPV2 and measured changes in calcium uptake. In calcium-imaging experiments using the wild type hTRPV2, we reconfirmed inhibition of calcium influx by PL ( $p \leq 0.0001$; two-way ANOVA) when cannabidiol was evoked (Figure 2e,f, Figures S8-S10). The Arg539Ala mutation showed a loss of inhibition by PL $(p \leq 0.0001$; two-way ANOVA), consistent with its predicted role as a PL binding site. More interestingly, the Thr522Met mutation not only showed a loss of inhibition by PL ( $p \leq 0.0001$; two-way ANOVA) but also overall increased calcium uptake both with and without the addition of PL. It is not possible to determine from these experiments whether this increase in calcium uptake was due to increased hTRPV2 expression and/or trafficking to the cell membrane or because the Thr520Met mutation increased channel activity in response to CBD. The Thr520Met mutation not only alters hydrogen bridging possibilities but also introduces a bulkier residue that could restrict access of PL to the binding site. Taken together, our data highlight the relevance of these two residues in the antagonism of TRPV2 by PL with changes in calcium influx.

Structurally, PL induces conformational changes in the TRPV2 selectivity filter and lower gate. Both remain closed when PL is bound; the conformation of the lower gate shifts slightly downward, and His651 rotates out of the pore relative to the recently published apo rat TRPV2 in nanodisc structure (PDB 6U84) (Figures 3a,b). The downward shift in the PLbound structure seems to originate at the S4-S5 linker, which shifts toward S5 by $4.0 \AA$ and downward by $2.9 \AA$, with the adjacent helices, including S6, shifting to compensate (Figure $3 c$ ). The shift of the S4-S5 linker toward S5 is reminiscent of the changes seen in the recent structure of rTRPV2 bound to $\mathrm{CBD}$, state 2 (PDB 6U88) (Figure S11), though that structure did not have the additional downward shift of the S4-S5 linker seen in the PL-bound structure. On top of this, the PL-bound structure has the $\mathrm{C}$-terminal domain wrapped around its $\beta$ sheet region paired with a downward pivot of the intracellular ankyrin repeat domains (Figure $3 \mathrm{~d}$ ), again as seen in the CBD state 2 structure (PDB 6U88) (Figure S11). Overall, the entire molecule shifts downward by $2.8 \AA$ relative to the central pore, creating a transient allosteric tunnel where PL sits (Figure $3 \mathrm{~d}$ ). The transmembrane domain of the PL-bound structure also aligns well to the apo truncated rabbit TRPV2 structure, which was predicted to be desensitized ${ }^{27}$ (Figure S11). Taken together, these changes suggest that PL may inhibit TRPV2 by immobilizing the S4-S5 linker and locking TRPV2 in a desensitized state.

hTRPV2 Expression Is Required by CNS Cancer Cell Lines and Associated with Disease Progression and Poor Prognosis in GBM. To probe the role of hTRPV2 on the antiproliferative effects of PL, we screened PL against the NCI-60 panel of cancer cell lines ${ }^{29}$ and analyzed the publicly available CTRP data. ${ }^{30}$ In both panels, cell lines expressing hTRPV2 were very sensitive to PL (Wilcoxon rank-sum test $p$ $<0.0001$ and 0.005 , respectively, for the CTRP and NCI-60 panels; Figure 4a, Figures S12-S14), supporting the importance of hTRPV2 modulation as a mechanism that contributes to the anticancer activity by PL. To identify cancer types potentially more vulnerable to hTRPV2 targeting, we analyzed CRISPR-Cas9 hTRPV2 loss-of-function screens performed in 764 cancer cell lines within the Cancer Dependency Map (DepMap). ${ }^{31}$ Cell lines from the central nervous system (CNS) were the most sensitive to hTRPV2 loss (Figure 4b, Figure S15), suggesting CNS tumors as candidate disease models for hTRPV2 targeting.

To further investigate the importance of hTRPV2 in different cancer types, we tested its prognostic value across 9785 tumor samples belonging to 30 cohorts from The Cancer Genome Atlas (TCGA; Table S4). High hTRPV2 expression is strongly associated with poor survival in patients with gliomas $\left(p=4.37 \times 10^{-13}\right.$, log-rank test; Figure S16), also when stratified into the low-grade glioma (LGG) ${ }^{32}$ and $\mathrm{GBM}^{33}$ cohorts individually ( $p=0.028$ and 0.0087 , respectively, logrank test; Figure $4 c$ ). The prognostic value of hTRPV2 was independently validated in two other cohorts of gliomas and GBMs from the Chinese Glioma Genome Atlas (CGGA, http://www.cgga.org.cn/; Figure S17). We also found a significant progressive increase of hTRPV2 expression with tumor grade (Figure 4d) and from primary to matched recurrent GBM samples (Figure 4e). These results thus associate hTRPV2 overexpression with worse outcome in GBM.

We next analyzed the transcriptomes of human GBM single cells $^{34}$ and found hTRPV2 expressed predominantly in endothelial and microglia/macrophage tumor cells but not in neoplastic tumor cells (Figure 4f, Figure S18) or normal brain samples $^{35}$ (Figure S19). Concordantly, hTRPV2 expression is negatively associated with tumor purity, i.e., with the number of neoplastic cells vs non-neoplastic cells present in the LGG and GBM samples (Spearman's correlation coefficient, $\rho=$ -0.45 and $-0.36, p=7.5 \times 10^{-25}$ and $3.1 \times 10^{-14}$, respectively; Figure S20), showing that hTRPV2 is mainly expressed in the tumor microenvironment. This was validated in selected samples from patients diagnosed with grade II-IV glioma, where immunostaining for hTRPV2 showed expression in glial and endothelial cells (Figure 4g, Figure S21). Most significantly, in areas of microvascular proliferation, a characteristic pathologic feature of GBM, endothelial cells showed a significant overexpression of hTRPV2 (Figure S22), and a distinct staining distribution/pattern (Figure 4g). In glial cells, staining was cytoplasmic and with a punctate pattern, whereas endothelial cells showed an intense and diffuse staining of the cytoplasmatic membrane (Figure $4 \mathrm{~g}$ ). Highermagnification imaging depicts a clear punctate staining in glial cells and diffuse membranous staining in the endothelial cells lining tumor vessels (Figure S22). This links with the fact that high hTRPV2 expression plays a role in angiogenesis ${ }^{36}$ and tumor progression and associates with poor prognosis (Figure $4 c-e)$. When we tested the prognostic value of hTRPV2 independent of the endothelial and microglia/macrophage content, we confirmed that high hTRPV2 expression was still significantly associated with prognosis in GBM (Cox proportional-hazards regression $p<0.05$ ) but not in low-grade glioma 
a

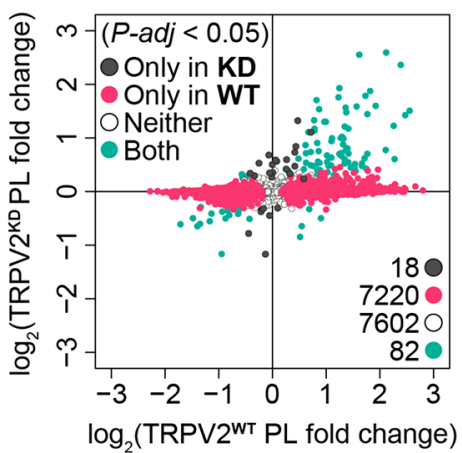

b

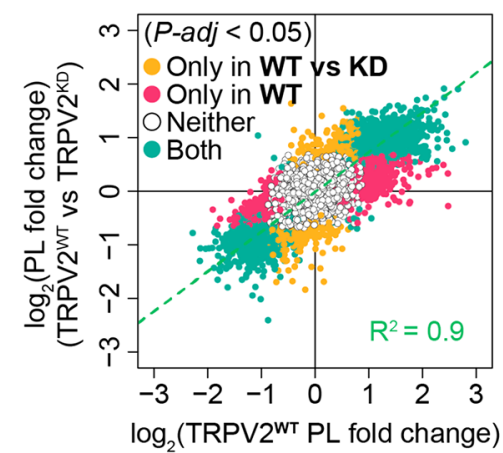

C

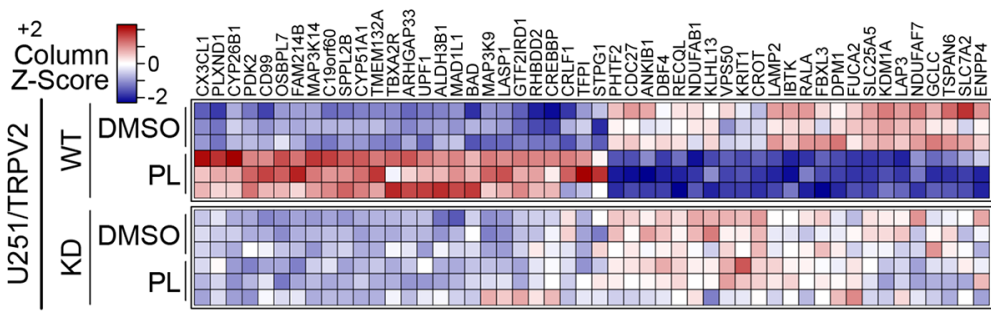

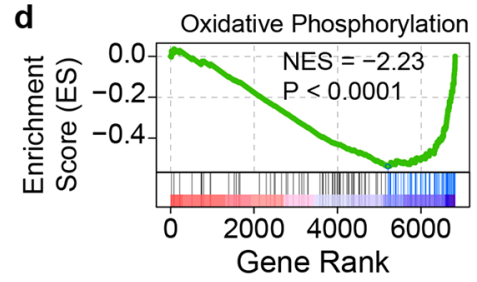

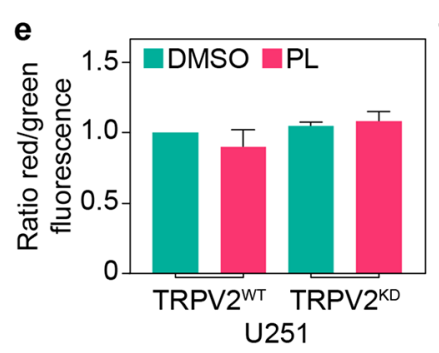

GO Mitochondrial Envelope
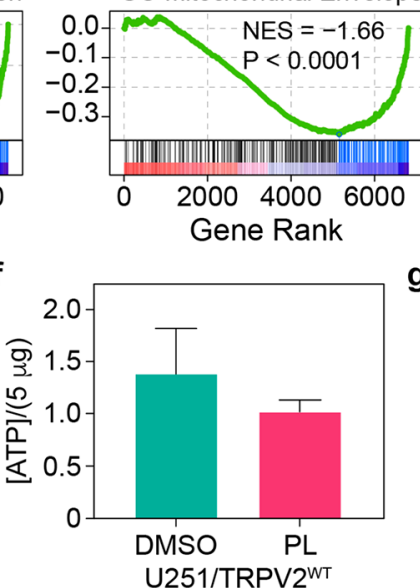

REACTOME Translation

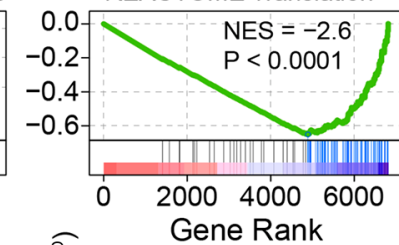

g

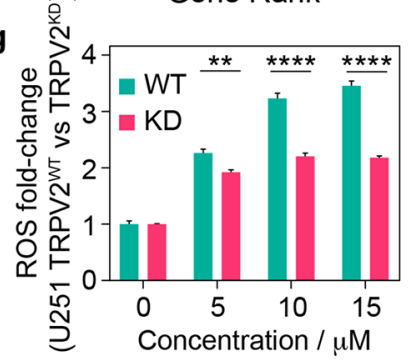

Figure 5. PL treatment affects ROS in hTRPV2 WT/KD U251 glioblastoma cells. (a) Transcriptomic (RNA-seq) analysis of PL effects on U251 $\mathrm{TRPV}^{\mathrm{WT}}$ vs TRPV ${ }^{\mathrm{KD}}$ cells. The log2 fold changes between WT and KD cells show loss of PL activity in the knock-down. The numbers of significant $\left(p_{\text {adj }}<0.05\right)$ genes are shown in the inset and are color annotated as indicated. (b) Log fold change correlation of genes between TRPV2 ${ }^{\mathrm{WT}}$-PL-treated and TRPV2 ${ }^{\mathrm{KD}}$ PL-treated U251 cells: genes are colored base on $p$-adjusted values as indicated in the inset, highlighting low correlation between the two data sets. (c) Heatmap of reads (FPKM; expressed as a Z-score for each column, as indicated in the inset) of the top 50 significantly $\left(p_{\text {adj }}<0.05\right)$ differentially expressed genes in PL-treated TRPV2 ${ }^{\mathrm{WT}}$ and TRPV2 ${ }^{\mathrm{KD}} \mathrm{U} 251$ cells. (d) GSEA analysis of TRPV2 ${ }^{\mathrm{WT}}$ U251 cells treated with PL identified downregulation of the Hallmark gene-set "Oxidative Phosphorylation", the gene-ontology "Mitochondrial Envelope", and the REACTOME "Translation" signatures. The plots show the running sum for the molecular signature database gene set within the TRPV2 ${ }^{\mathrm{WT}} / \mathrm{PL}$ data including the maximum enrichment score and the leading-edge subset of enriched genes. (e) Determination of mitochondrial potential using a fluorescent readout on a plate reader in PL-treated TRPV2 ${ }^{\mathrm{WT}}$ and TRPV2 ${ }^{\mathrm{KD}} \mathrm{U} 251$ cells determined following treatment with $10 \mu \mathrm{M}$ of the dye JC-1. The ratio of green $(\lambda=530 \pm 15 \mathrm{~nm})$ and red/orange $(\lambda=590 \pm 17.5 \mathrm{~nm})$ fluorescence emission was monitored after $48 \mathrm{~h}$ of PL treatment. Data represent the mean \pm SD $(n=3)$. (f) ATP content determination using a luciferase-based ATP detection assay following $48 \mathrm{~h}$ of PL treatment of TRPV2 ${ }^{\mathrm{WT}} \mathrm{U} 251$ cells. Data represent the mean $\pm \mathrm{SD}(n=5)$. (g) PL-induced ROS production is TRPV2-dependent $(n=3$, two-way ANOVA, Sidack's multiple comparisons test $* * p=0.0056, * * * *<0.001)$. Lower levels of ROS were observed in the U251-TRPV2-KD cell line compared to the control cell line. Cellular ROS levels were measured after $1 \mathrm{~h}$ of PL treatment and quantified with flow cytometry.

$(p=0.5)$. Altogether, our data and others ${ }^{37}$ support GBM as a candidate disease for treatment with hTRPV2 ligands, including PL.

hTRPV2 Knockdown in Glioblastoma Cells Reduces Sensitivity to PL and ROS Production. We first tested the effect of PL in the viability of different GBM cell lines (U251, U87, YKG1, and D8TRG/06MG) which express significant amounts of hTRPV2 (Figure S23 and Table S5). These cell lines were sensitive to $\mathrm{PL}$ (with $\mathrm{IC}_{50}$ values of 3.0, 6.6, 4.7, and $6.6 \mu \mathrm{M}$, respectively) after $72 \mathrm{~h}$. Endothelial brain cells, which also express hTRPV2, were sensitive to PL $\left(\mathrm{IC}_{50}=1.6 \mu \mathrm{M}\right)$, while both microglia and astrocytes were less sensitive $\left(\mathrm{IC}_{50}>\right.$ $10 \mu \mathrm{M}$ ) (Figure S23 and Table S5). Despite the high hTRPV2 expression found in the tumor microenvironment, the high sensitivity to PL of cell lines expressing hTRPV2 and high hTRPV2 dependency of CNS cell lines support the use of GBM cell line models to study the relationship between hTRPV2 and PL treatment. Our data show that cells expressing hTRPV2 are sensitive to PL. 


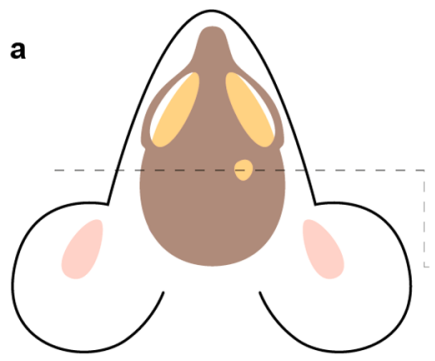

Make burr hole in skull
Inject GBM cells

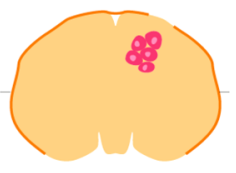

Induce tumor

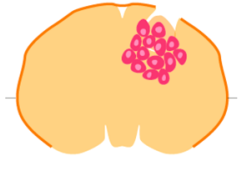

Make a cavity

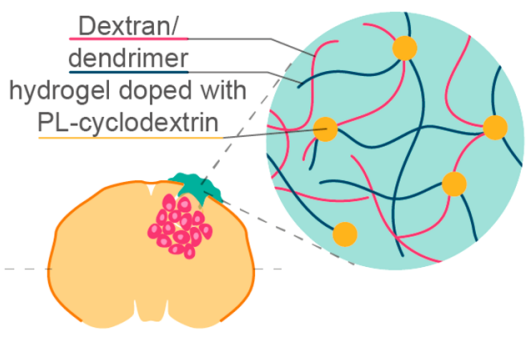

Implant hydrogel

Trial end

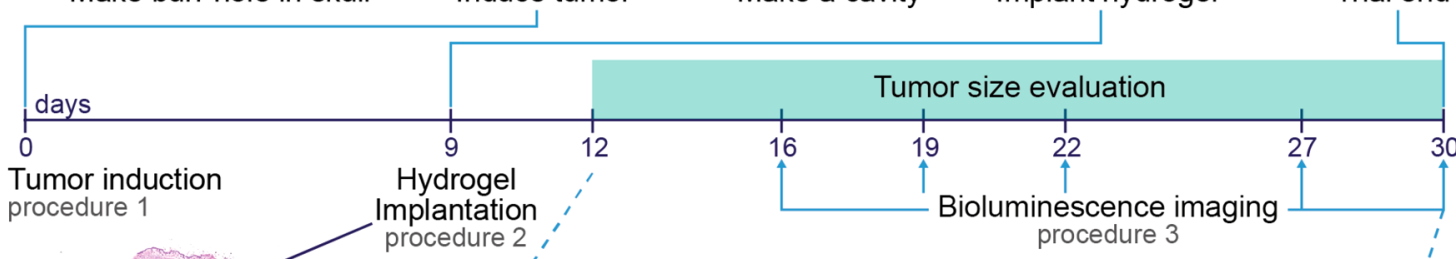

b

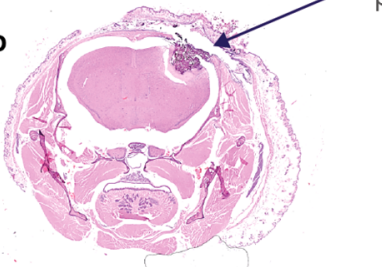

d U251 model
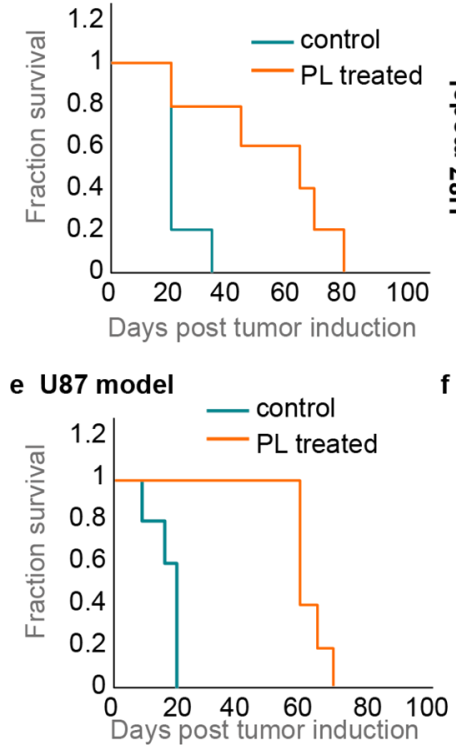

\section{c}

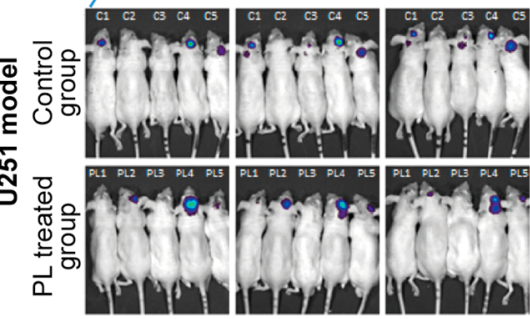

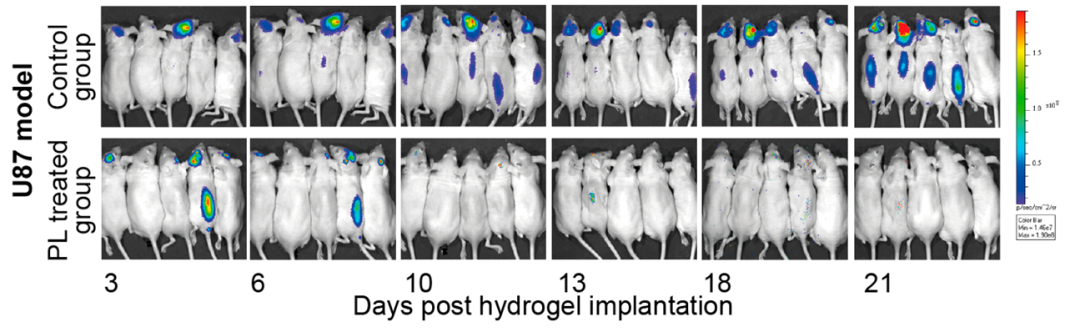

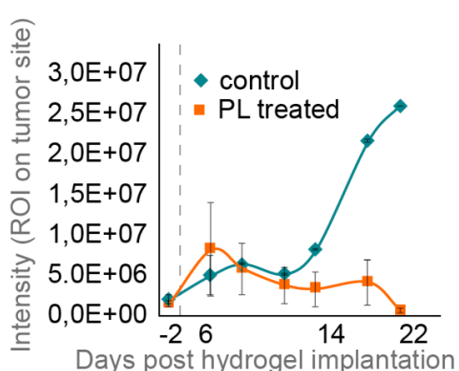

$\mathbf{g}$

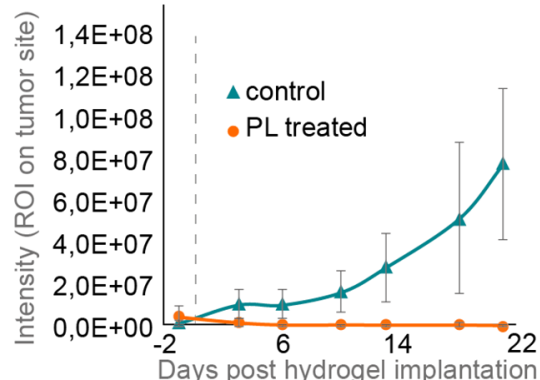

Figure 6. PL-loaded hydrogel scaffolds treat glioblastoma multiforme in vivo. (a) Experiment design showing the development of a biocompatible, PL-loaded hydrogel suitable for local treatment of GBM. Experiment flow: U251 or U87-MG cells were injected intracranially ( $n=5$ per group). 8 days later, engraftment was confirmed with bioluminescent imaging. On day 9, PL-loaded or unloaded hydrogels were implanted. Tumor growth was assessed at regular intervals by using bioluminescent imaging. (b) Histological image of a full mouse brain showing the hydrogel implant. (c) Bioluminescent images of U251 and U87-MG xenograft mice treated with PL-loaded hydrogel (50 mg/kg) or unloaded hydrogel (control). Data show that PL treatment successfully reduces tumor volume over a period of 21 days relative to the control, in which the tumor grows exponentially, on both models of GBM. Tumor burden in mice treated with PL-loaded or unloaded hydrogel as measured by luciferase activity for (f) U251 and (g) U87-MG models. Data are expressed as luciferase intensity (ROI on tumor site) as an indication of average tumor load in the control group at each time point and represent group mean \pm SEM. Data show a significant tumor burden difference between both groups $(p \leq 0.01, n=5$, MannWhitney test) for (f) U251 and (g) U87-MG models. The Kaplan-Meier survival curve shows that mice treated with PL-loaded hydrogels have significantly increased survival ( $p \leq 0.001$, log-rank Mantel-Cox test) relative to the control group for (h) U251 and (i) U87-MG models. The experiment cutoff criteria included an increase in tumor size of around 500\% relative to the original size measured for each mouse prior to hydrogel implantation.

To investigate the relationship between hTRPV2 and PL treatment, we knocked down the expression of hTRPV2 (by $82 \pm 12 \%$, see Figure S24) in the U251 glioblastoma cells (TRPV2 ${ }^{\mathrm{KD}}$ ) using the CRISPRi technology with dCas9-KRABguide RNA plasmids. ${ }^{38}$ We found that the PL-induced effect on viability is diminished when hTRPV2 expression is reduced as shown in Figure S25a-c. TRPV2 ${ }^{\mathrm{KD}}$ cells are 2-fold less sensitive to the antiproliferative effects of PL $(p=0.0002$, unpaired $t$-test, Figure S25a). We then performed RNAsequencing analysis of U251 cells (TRPV2 ${ }^{\mathrm{WT}}$ or TRPV2 $2^{\mathrm{KD}}$ ) 
treated with PL for 48 h. Principal component analysis confirmed a stronger effect of PL-treatment in TRPV2 ${ }^{\mathrm{WT}}$ cells, with little separation between control and treatment conditions in TRPV $2^{\mathrm{KD}}$ cells (Figure S25d) confirming successful knockdown of hTRPV2. Indeed, comparison of the fold changes (PL-treated vs DMSO control) between TRPV2 ${ }^{\mathrm{WT}}$ and TRPV $2^{\mathrm{KD}}$ cells identified only 100 genes that were significantly $\left(p_{\text {adj }}<0.05\right)$ differentially expressed in the knockdown as opposed to 7302 genes in the case of TRPV2 ${ }^{\mathrm{WT}}$ cells (Figure 5a). This was further confirmed when comparing the fold changes introduced by PL in TRPV2 ${ }^{\mathrm{WT}}$ vs TRPV2 ${ }^{\mathrm{KD}}$ cells, which yielded high correlation to PL-treated TRPV2 ${ }^{\mathrm{WT}}$ cells only (Figure 5b). This shows that hTRPV2 knockdown in glioblastoma cells reduces the sensitivity to PL and its induced transcriptomic changes.

Inspection of significantly differentially expressed genes upon PL treatment $\left(p_{\text {adj }}<0.05\right.$ and absolute fold-change $>1.5$ ) identified several candidates linked to the electron transport chain as well as in trafficking of ATP (including NDUFAB1, NDUFAF7, KDM1A, and SLC25A5, Figure 5c). Indeed, geneset enrichment analysis (GSEA) highlighted the downregulation of oxidative phosphorylation as well as that of several mitochondrial signatures, including that of the mitochondrial envelope (Figure 5d, Figure S25e,f). To validate this observation, we asked if the mitochondrial membrane potential was compromised following treatment with PL. Using the cationic dye JC-1 that changes its fluorescent signal according with the energy state of the mitochondria, we interrogated mitochondrial depolarization and found a decrease in TRPV2 ${ }^{\mathrm{WT}} \mathrm{U} 251$ cells treated with PL, which was not observed in TRPV2 ${ }^{\mathrm{KD}} \mathrm{U} 251$ cells (Figure 5e). In agreement with this observation, ATP content was reduced following PL treatment of U251 cells compared to a negative control (Figure 5f).

$\mathrm{PL}$ is known to cause an increase in ROS, which ultimately leads to cell death; however, the mechanism by which this occurs has not previously been associated with TRPV2. ${ }^{39} \mathrm{We}$ found that U251-TRPV2-KD cells treated with PL accumulate significantly less ROS relative to the control cells with normal hTRPV2 expression levels (Figure $5 \mathrm{~g}$ ). This was further supported by rescue studies using $N$-acetyl-L-cysteine (NAC). NAC is commonly used to identify and test ROS inducers and to inhibit ROS. Indeed, NAC was able to rescue U251 and U87MG cell viability in the presence of PL (Figure S26). This shows that NAC interfered with the activity of PL, further supporting that PL affects mitochondrial dysfunction. This implies that hTRPV2 plays a key role in the formation of ROS in cells after PL treatment.

Taken together, these observations suggest that PL treatment results in a TRPV2-dependent decrease of mitochondrial membrane potential, which ultimately leads to the loss of cellular ATP content. A recent study demonstrated that the increase of cellular ROS by defective mitochondria links to loss of translation ${ }^{40}$ in agreement with our transcriptomic analysis, which identified strong downregulation of translational signatures (Figure 5d).

Treatment of GBM via Sustained and Local Implanted PL-Scaffolds. Having obtained promising in vitro functional assay data and established a mode of action in a preclinical scenario, we next explored the therapeutic potential of PL in a murine orthotopic xenograft model of GBM (Figure $6 \mathrm{a}, \mathrm{b})$. In our experience, PL was poorly soluble in biocompatible formulations, and subcutaneous injections of
$50 \mathrm{mg} / \mathrm{kg}$ resulted in toxicity with limited effects on intracranial tumor growth (Figure S27). After considering these results, we sought out a more effective mode for in vivo drug delivery. Thus, we encapsulated PL in $\beta$-cyclodextrin (1:2 ratio), which dramatically improved its aqueous solubility. Furthermore, to enhance therapeutic efficacy, we aspired to eliminate the challenge of crossing the blood-brain barrier and create a therapy suitable for local and sustained drug administration. To this end, we devised implantable dextrandendrimer hydrogel scaffolds ${ }^{41-44}$ that were doped with PL encapsulated in $\beta$-cyclodextrin. The cyclodextrin was used as a "carrier" molecule to facilitate the dissolution of PL. $\beta$ Cyclodextrin is a cyclic oligosaccharide, which consists of a macrocyclic ring of 7 glucose subunits with a hydrophobic interior and hydrophilic exterior that forms a complex with the hydrophobic PL compound. This new material allowed us to obtain a high concentration of PL at the tumor site. Moreover, we minimized leakage of PL to healthy tissue by decorating the hydrogel with aldehyde groups from oxidized dextran to interact with cancer tissue amines and form adhesive bonds, as previously reported. ${ }^{41-44}$ Profiling of our drug delivery system showed a significant discharge of PL in the first $4 \mathrm{~h}$ followed by steady release (3\% of total) for at least $192 \mathrm{~h}$ under physiological conditions (Figure S28). These data provide a rationale to the in vitro toxicity experiments in which PL-doped hydrogels were able to induce near complete cell death after incubation with U251 cells for $24 \mathrm{~h}$. The empty hydrogels did not affect cell viability (Figure S29).

We proceeded to study the in vivo therapeutic efficacy of the PL-doped hydrogels in two murine models of GBM (U251 and U87-MG). The U251 malignant glioma cell line is known to mimic the salient features of human GBM and displays similarities at the genetic level. ${ }^{45,46}$ The U87 GBM model is commonly used for assessing tumor angiogenesis and antiangiogenic therapies in GBM. ${ }^{46,47}$ Tumors were induced through direct injection of $2.5 \times 10^{5} \mathrm{U} 251$-GFP-luc or $2.5 \times$ $10^{5}$ U87-MG-luc cells into the brain of athymic nude mice (Crl:NU(NCr)-Foxn $1^{\text {nu }}$ ) via a burr hole $2.5 \mathrm{~mm}$ lateral and 1.5 $\mathrm{mm}$ posterior to bregma, above the right cerebrum. Nine days post-tumor-injection, hydrogel scaffolds loaded with PL (50 $\mathrm{mg} / \mathrm{kg}$ ) were implanted in the supratentorial region directly overlaying the area where the tumor was implanted in the right hemisphere of the brain (Figure 6a,b). Empty hydrogel implants, i.e., lacking the PL component, were used as controls. Tumor volume was measured over time by bioluminescence with Xenogen IVIS Lumina for 21 days after hydrogel implantation (Figure 6c). The difference in survival between the two groups was highly significant $(p \leq$ 0.001, log-rank Mantel-Cox test) for both U251 (Figure 6d) and U87 (Figure 6e) models. The mice exposed to PL-doped hydrogel showed significant tumor size reduction at the end of the study ( $n=5, p \leq 0.01$ Mann-Whitney test) for both U251 (Figure 6f) and U87 (Figure 6g) models. To further demonstrate the superiority of the local PL-hydrogel scaffold, the efficacy of the local delivery was compared with systemic (subcutaneous) injection of PL alone, both at a final concentration of $50 \mathrm{mg} / \mathrm{kg}$ PL (Figure S27). We found that although local administration of PL by means of a hydrogel scaffold resulted in almost complete remission of the GBM tumor, no effect on tumor reduction was attained following systemic administration of the same concentration of PL. These results provide substantial evidence that the hydrogel scaffold is essential for higher therapeutic performance. In 

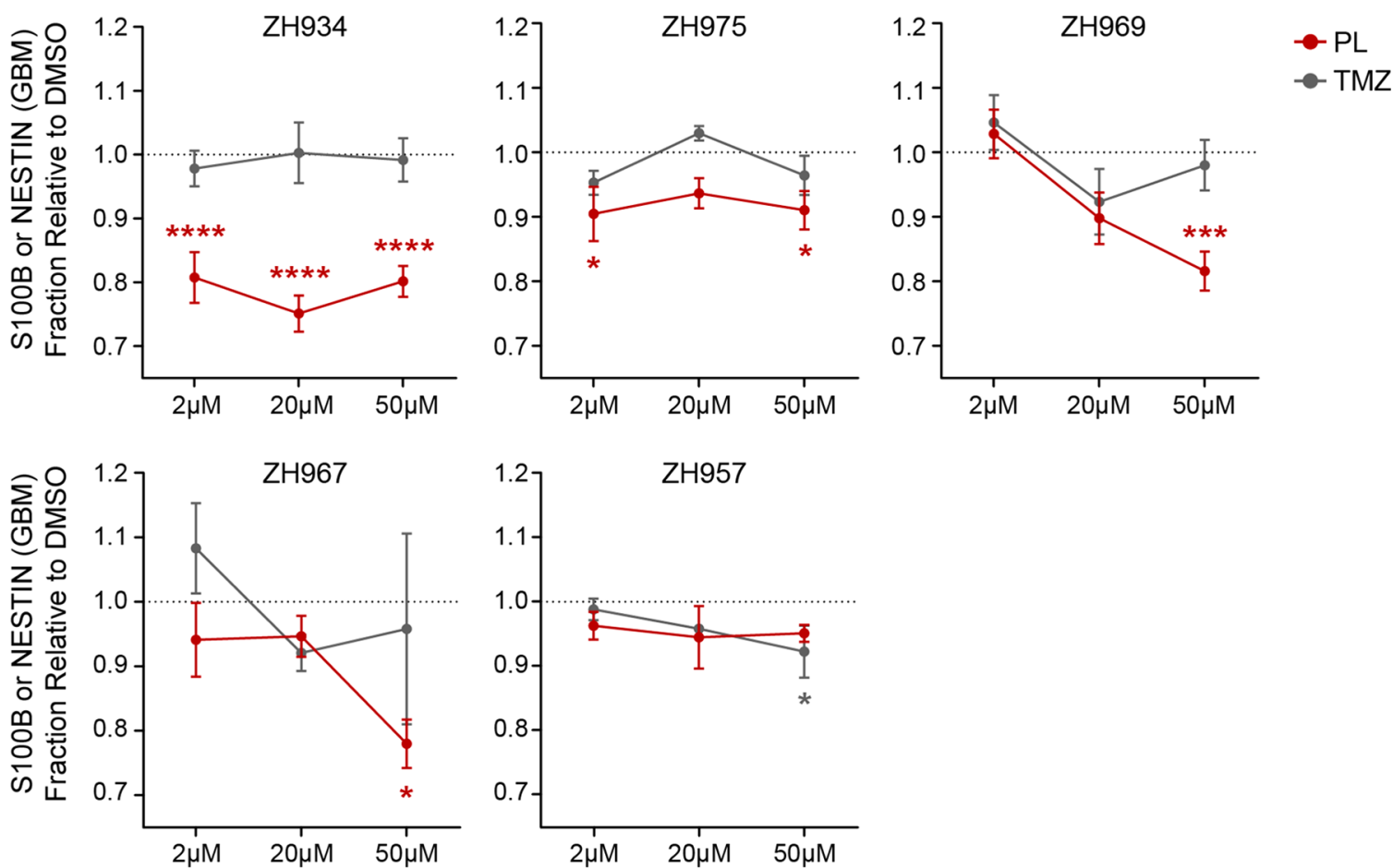

Figure 7. Ex vivo drug response of GBM cells derived from five patients. In this experiment, the $\mathrm{ZH}$ prefix is followed by the unique patient ID ( $n=$ 5 ). Colored lines represent the tested drugs; piperlongumine (PL, red) and temozolomide (TMZ, gray), where the $x$-axis corresponds to the concentrations tested $(2,20,50 \mu \mathrm{M})$. The $y$-axis denotes fraction reduction of S100B+CD45- or NESTIN+CD45- cells identified by immunofluorescence relative to the DMSO control $(50 \mu \mathrm{M})$. Values plotted as mean \pm SEM. Two-way ANOVA followed by Dunnett's multiple comparisons test was performed to calculate statistical significance $(n=4-5$ replicate wells per treatment condition, $n=15$ wells for DMSO control). $* p_{\text {adj }}<0.05, * * p_{\text {adj }}<0.01, * * * p_{\text {adj }}<0.001, * * * * p_{\text {adj }}<0.0001$.

addition, no apparent signs of disease (not related to local tumor growth) were seen in any of the animals for 21 days after hydrogel implantation as indicated by the preservation of steady body weight (Figure S30), pointing at both drug and hydrogel biocompatibility. Histopathology of the liver, kidney, lung, spleen, gastrointestinal tract, and heart showed no significant changes or evidence of toxicity of PL. No evidence of necrosis or other lesions (e.g., inflammation) was seen in the brain, at the site of implantation (Figure S31).

Monitoring of tumor growth in the brain of PL-treated mice showed delayed tumor progression (Figure $6 \mathrm{~d}-\mathrm{g}$ ). Leptomeningeal dissemination was seen both through in vivo imaging, as a second and less intense bioluminescence signal, dorsal and caudal to the primary tumor and overlaying the posterior part of the brain and spinal cord (Figure 6c), and also microscopically as tumor cells infiltrating and expanding the leptomeninges. Meningeal bioluminescence was seen in $60 \%$ (for U251 model) and $80 \%$ (for U87 model) of the mice in the control groups, and only $20 \%$ for both U251 and U87 models in the PL-treated group. PL-treated mice showed reduced tumor growth at the site of injection, and tumor cells were rarely found to infiltrate the meninges, as depicted in Figure $6 \mathrm{c}$ and in Figure S32. This suggests that treatment with PL may mitigate GBM proliferation and invasion. Immunohistochemistry showed that hTRPV2 is indeed expressed in the tumor xenografts with higher expression in the PL-treated groups (Figure S33). Altogether, the in vivo therapy using local scaffolds to deliver PL was highly effective in both models of GBM, presenting an almost complete tumor abrogation, associated with a higher survival rate for as long as 80 days for the U251 model and 70 days for U87 model.
PL Induces Selective ex Vivo Response in GBM Patient-Derived Cells. Next, we evaluated the clinical potential of PL using Pharmacoscopy, a technology that utilizes high content automated microscopy to measure ex vivo drug response directly in patient-derived cells. ${ }^{48,49}$ The relevance of Pharmacoscopy has been previously demonstrated in a clinical trial for aggressive hematological malignancies, where it was used to identify the best treatment option for patients, ultimately leading to better outcomes such as increased progression-free-survival. ${ }^{48}$ Here, we tested PL ex vivo drug responses in five surgically derived GBM patient samples at three different drug concentrations. Freshly dissociated cells that were initially cryopreserved before the assay were incubated with PL, standard-of-care temozolomide (TMZ), or DMSO as a negative control for $48 \mathrm{~h}$. Then, to identify potentially malignant cells, dissociated cells were immunofluorescently labeled with the astrocyte marker S100B as well as NESTIN (Figure 7), a marker for proliferating neural progenitors' cells, while CD45 (Figure S34) was included as a counterstain to identify nonmalignant immune cells. In four out of five tested patient samples (80\%), PL induced a significant decrease in the fraction of glioblastoma cells (defined inclusively as either S100B or NESTIN positive cells) at the highest tested concentration $(50 \mu \mathrm{M})$ compared to the negative control. Among these four responders, two patient samples also responded to PL at lower concentrations. The observed effect was "on-target"-preferentially affecting the putatively malignant cells and not the immune populations, which was also reflected in our relative fraction readout. Furthermore, when compared to TMZ, which is usually used at higher in vitro concentrations than the ones tested, PL 
exerted a stronger effect within this range of low doses (Figure 7, Figure S34). Taken together, we provide promising evidence of PL-induced selective drug response in tumor samples derived from GBM patients. As our tested cohort is relatively small, we hope future studies will further investigate the clinical potential that PL might hold as a putative GBM therapy.

\section{CONCLUSIONS}

Statistical learning may be used to accelerate drug discovery programs by prioritizing biochemical screens; such methods ought to be generally applicable to molecules of synthetic and natural origins. Under such a concept, our computer-driven and disease agnostic approach constitutes also a viable alternative to swiftly repurpose chemical matter and motivate the development of innovative therapeutics. Here, by dissecting the chemical features of PL, a hitherto unknown relationship to hTRPV2 emerged. The mechanistic insights for hTRPV2 have been rare, and its (patho)biological relevance is largely unknown. ${ }^{50,51}$ To further our understanding of the hTRPV2 mechanism of modulation by exogenous compounds, we determined the PL-bound full-length rat TRPV2 structure at an overall resolution of $3.5 \AA$ and identified a transient, allosteric binding site for PL along the S4-S5 linker of the channel. A high-quality open state of TRPV2 has not yet been resolved, so it is difficult to predict the exact mechanism of PL inhibition of channel opening. However, the S4-S5 linker plays a key role in channel opening across the entire TRP channel family, ${ }^{52}$ which suggests that small molecule ligands for this region modulate function. Calcium imaging data of hTRPV2 mutants with the addition of PL further established the importance of the Arg539 and Thr522 residues in TRPV2 channel's calcium influx modulation and highlighted the role of Arg539 against PL antagonism for TRPV2, consistent with the cryo-EM structure.

Importantly, we establish that modulation of hTRPV2 by PL affords a biologically relevant phenotype, in particular against GBM in which hTRPV2 overexpression correlates with tumor grade, and that the anticancer activity of PL is high in cell lines with high hTRPV2 gene expression, which is required by CNS cancer cell lines. Moreover, the CRISPRi-induced downregulation of hTRPV2 showed that GBM cells treated with PL accumulate significantly less ROS relative to the control cells and that knockdown of hTRPV2 also decreases sensitivity to PL, which suggests the importance of TRPV2 for the anticancer activity of PL. Indeed, with the presence of damaged mitochondria (decreased mitochondrial membrane potential and ATP content) in hTRPV2 ${ }^{\mathrm{WT}}$ treated with PL, increased ROS fold is expected in these cells. These increased ROS levels impinge upon nuclear transcription of mitochondrial targeted proteins, as observed in the RNaseq analysis. Finally, we also showed that hTRPV2 is a marker of poor prognosis for GBM patients, and immunohistochemistry of GBM tissues samples showed a strong correlation between hTRPV2 overexpression and aggressiveness of the disease.

GBM remains an unmet medical need, with newly diagnosed patients having a life expectancy of only 11-15 months (which has not improved in the last 30 years), and new therapeutics are urgently sought after. To that end, formulation of PL in a hydrogel for sustained intracranial release afforded an efficacious GBM treatment in mice using two orthotopic GBM models to harness an innovative mechanism of action. This treatment resulted in a highly significant decrease in disease progression and increase in survival, which greatly contributes to the avoidance of relapses of GBM. Furthermore, using Pharmacoscopy, we have demonstrated that PL shows promising on-target ex vivo responses in surgically derived GBM patient samples, further strengthening the clinical potential and applicability of our findings. Altogether, supported by functional and structural data, we showed the power of computer perception and decision making from complex data patterns and expect it to play an increasing role in future drug discovery and in the success of GBM therapy.

\section{ASSOCIATED CONTENT}

\section{(s) Supporting Information}

The Supporting Information is available free of charge at https://pubs.acs.org/doi/10.1021/acscentsci.1c00070.

Materials and methods; detailed characterization of small molecules; and additional data and figures (PDF) NMR, HPLC, and mass spectrometry data for the cis analogue (PDF)

NMR, HPLC, and mass spectrometry data for the trans analogue (PDF)

Model validation report (PDF)

GBM patient clinical annotations (XLSX)

\section{Accession Codes}

The cryo-EM density map and atomic coordinates of PLbound rat TRPV2 are deposited into the Electron Microscopy Data Bank and Protein Data Bank under accession codes EMD-21705 and PDB 6WKN.

\section{AUTHOR INFORMATION}

\section{Corresponding Authors}

Vera Y. Moiseenkova-Bell - Department of Systems Pharmacology and Translational Therapeutics, Perelman School of Medicine, University of Pennsylvania, Philadelphia, Pennsylvania 19104, United States; Email: vmb@ pennmedicine.upenn.edu

Gonçalo J. L. Bernardes - Instituto de Medicina Molecular João Lobo Antunes, Faculdade de Medicina, Universidade de Lisboa, 1649-028 Lisboa, Portugal; Yusuf Hamied Department of Chemistry, University of Cambridge, CB2 $1 E W$ Cambridge, United Kingdom; (1) orcid.org/00000001-6594-8917; Email: gb453@cam.ac.uk, gbernardes@ medicina.ulisboa.pt

\section{Authors}

João Conde - Instituto de Medicina Molecular João Lobo Antunes, Faculdade de Medicina, Universidade de Lisboa, 1649-028 Lisboa, Portugal; 이이이.org/0000-0001-84226792

Ruth A. Pumroy - Department of Systems Pharmacology and Translational Therapeutics, Perelman School of Medicine, University of Pennsylvania, Philadelphia, Pennsylvania 19104, United States

Charlotte Baker - Instituto de Medicina Molecular João Lobo Antunes, Faculdade de Medicina, Universidade de Lisboa 1649-028 Lisboa, Portugal

Tiago Rodrigues - Instituto de Medicina Molecular João Lobo Antunes, Faculdade de Medicina, Universidade de Lisboa, 1649-028 Lisboa, Portugal

Ana Guerreiro - Instituto de Medicina Molecular João Lobo Antunes, Faculdade de Medicina, Universidade de Lisboa, 1649-028 Lisboa, Portugal 
Bárbara B. Sousa - Instituto de Medicina Molecular João Lobo Antunes, Faculdade de Medicina, Universidade de Lisboa, 1649-028 Lisboa, Portugal

Marta C. Marques - Instituto de Medicina Molecular João Lobo Antunes, Faculdade de Medicina, Universidade de Lisboa, 1649-028 Lisboa, Portugal

Bernardo P. de Almeida - Instituto de Medicina Molecular João Lobo Antunes, Faculdade de Medicina, Universidade de Lisboa, 1649-028 Lisboa, Portugal

Sohyon Lee - Institute of Molecular Systems Biology, ETH Zürich, 8093 Zürich, Switzerland

Elvira P. Leites - Instituto de Medicina Molecular João Lobo Antunes, Faculdade de Medicina, Universidade de Lisboa, 1649-028 Lisboa, Portugal

Daniel Picard - Department of Pediatric NeuroOncogenomics, DKFZ, Heidelberg 69120, Germany; Department of Pediatric Neuro-Oncogenomics, DKTK, Essen D-45147, Germany; Department of Pediatric Oncology, Hematology, and Clinical Immunology, Medical Faculty, University Hospital Düsseldorf, Düsseldorf 40225, Germany

Amrita Samanta - Department of Systems Pharmacology and Translational Therapeutics, Perelman School of Medicine, University of Pennsylvania, Philadelphia, Pennsylvania 19104, United States

Sandra H. Vaz - Instituto de Medicina Molecular João Lobo Antunes, Faculdade de Medicina, Universidade de Lisboa, 1649-028 Lisboa, Portugal; 이이. org/0000-0003-42589397

Florian Sieglitz - Instituto de Medicina Molecular João Lobo Antunes, Faculdade de Medicina, Universidade de Lisboa, 1649-028 Lisboa, Portugal

Maike Langini - Department of Pediatric NeuroOncogenomics, DKFZ, Heidelberg 69120, Germany; Department of Pediatric Neuro-Oncogenomics, DKTK, Essen D-45147, Germany; Department of Pediatric Oncology, Hematology, and Clinical Immunology, Medical Faculty, University Hospital Düsseldorf, Düsseldorf 40225, Germany

Marc Remke - Department of Pediatric Neuro-Oncogenomics, DKFZ, Heidelberg 69120, Germany; Department of Pediatric Neuro-Oncogenomics, DKTK, Essen D-45147, Germany; Department of Pediatric Oncology, Hematology, and Clinical Immunology, Medical Faculty, University Hospital Düsseldorf, Düsseldorf 40225, Germany

Rafael Roque - Laboratório de Neuropatologia, Hospital de Santa Maria, Centro Hospitalar Universitário Lisboa Norte (CHLN) EPE, 1649-028 Lisboa, Portugal

Tobias Weiss - Department of Neurology and Brain Tumour Center, University Hospital Zürich and University of Zurich, 8091 Zürich, Switzerland

Michael Weller - Department of Neurology and Brain Tumour Center, University Hospital Zürich and University of Zurich, 8091 Zürich, Switzerland

Yuhang Liu - Discovery Sciences, Worldwide Research and Development, Pfizer Inc., Groton, Connecticut 06340, United States

Seungil Han - Discovery Sciences, Worldwide Research and Development, Pfizer Inc., Groton, Connecticut 06340, United States

Francisco Corzana - Departamento de Química, Universidad de La Rioja, 26006 Logroño, Spain; (1) orcid.org/00000001-5597-8127
Vanessa A. Morais - Instituto de Medicina Molecular João Lobo Antunes, Faculdade de Medicina, Universidade de Lisboa, 1649-028 Lisboa, Portugal

Cláudia C. Faria - Instituto de Medicina Molecular João Lobo Antunes, Faculdade de Medicina, Universidade de Lisboa, 1649-028 Lisboa, Portugal; Department of Neurosurgery, Hospital de Santa Maria, Centro Hospitalar Universitário Lisboa Norte (CHULN) EPE, 1649-028 Lisboa, Portugal

Tânia Carvalho - Instituto de Medicina Molecular João Lobo Antunes, Faculdade de Medicina, Universidade de Lisboa, 1649-028 Lisboa, Portugal

Panagis Filippakopoulos - Structural Genomics Consortium, Oxford University, OX3 7DQ Oxford, United Kingdom

Berend Snijder - Institute of Molecular Systems Biology, ETH Zürich, 8093 Zürich, Switzerland

Nuno L. Barbosa-Morais - Instituto de Medicina Molecular João Lobo Antunes, Faculdade de Medicina, Universidade de Lisboa, 1649-028 Lisboa, Portugal

Complete contact information is available at:

https://pubs.acs.org/10.1021/acscentsci.1c00070

\section{Author Contributions}

$\triangle^{\triangle}$ J.C., R.A.P., C.B., and T.R. contributed equally. J.C. designed the hydrogel scaffold and in vivo delivery strategy, and C.B. and J.C. performed in vivo experiments. T.R. designed and performed cheminformatics studies and analyzed data. C.B., A.G., B.B.S., M.C.M., F.S., E.P.L., A.S., and M.C.M. conducted biochemical and cell biology studies, which included $\mathrm{Ca}^{2+}$ flux assay measurements with the assistance of S.H.V., protein purification, sample preparation, and data analysis and interpretation. R.A.P., A.S., and V.Y.M.-B. conducted cryoEM studies and analyzed and interpreted data with the assistance of Y.L. and S.H. B.P.d.A. and N.L.B.-M. designed and performed bioinformatics analyses of TCGA, CTRP, NCI60, DepMap, and single-cell transcriptomic data. M.L. acquired mass spectrometry data. M.L. and D.P. interpreted and analyzed mass spectrometry, and M.R. supervised the work performed by M.L. and D.P. F.C. performed preliminary molecular dynamic simulations and DFT minimizations. P.F. interpreted and analyzed RNA sequencing data. T.C. performed the histopathological analysis of mouse xenographs and TRPV2 immunohistochemistry scoring. R.R. performed histopathological analysis of human tumors and TRPV2 immunohistochemistry scoring. S.L. and B.S. performed Pharmacoscopy experiments, and human tumor samples used were surgically removed and analyzed by T.W. and M.W. J.C., R.A.P., C.B., T.R., P.F., V.Y.M.-B., and G.J.L.B. wrote the manuscript with contributions from the remaining authors. G.J.L.B. conceived the research. V.Y.M.-B. and G.J.L.B. supervised the research. All authors agreed on the final version of the manuscript

\section{Notes}

The authors declare the following competing financial interest(s): J.C., C.B., T.R., and G.J.L.B. are coinventors on a patent application (WO2019/054891 A2) that incorporates discoveries described in this manuscript. T.R., J.C., and G.J.L.B. are cofounders and shareholders of Targ.Tex S.A., a company that develops TRPV2 antagonists for the treatment of cancer. 


\section{ACKNOWLEDGMENTS}

We thank Sabine Baxter for assistance with hybridoma and cell culture at the University of Pennsylvania Perelman School of Medicine Cell Center Services Facility. We acknowledge the use of cryo-EM instruments at Pfizer Inc. The cryo-EM work was supported by a grant from the National Institute of Health (R01GM103899 and R01GM129357 to V.Y.M.-B.). The TRPV2-flag-RFP construct cloned into the pEGFP-N2 vector was kindly provided by Prof. Kojima (Gunma University, Japan). The Developmental Therapeutics Program is acknowledged for screening PL against the NCI-60 cancer cell line panel. We are grateful to Carlos Custódia, Eunice Paisana, and Dr. João Barata for their assistance with cell-line modulation and the GBM tumor model, and to Dr. Vikki Cantrill for her assistance with the editing of this manuscript. Figure design by Claudia Flandoli (draw.science). The Histology and Comparative Pathology Laboratory of the Instituto de Medicina Molecular is acknowledged for technical support. We also thank FCT Portugal (FCT Investigator IF/00624/2015 to G.J.L.B., IF/01693/2014 to V.A.M. and IF/00595/2014 to N.L.B.-M., CEECIND/00436/2018 to N.L.B.-M., PhD studentship SFRH/BD/143583/2019 to B.B.S., PhD studentship PD/BD/128289/2017 to E.P.L., Postdoctoral fellowship SFRH/BPD/1187731/2016 to M.C.M.), Agencia Estatal Investigación of Spain (AEI, RTI-2018-099592-B-C21 to F.C.), EMBO (Installation Grant 3057 to N.L.B.-M.), and the Medical Research Council (MR/N010051/1 to P.F.). This project has received funding from the European Union's Horizon 2020 research and innovation programme under grant agreement $630731,675007,807281$, and 743640 . G.J.L.B. is a Royal Society University Research Fellow (URF $\backslash R \backslash 180019$ ).

\section{REFERENCES}

(1) Clemons, P. A.; et al. Small molecules of different origins have distinct distributions of structural complexity that correlate with protein-binding profiles. Proc. Natl. Acad. Sci. U. S. A. 2010, 107, 18787-18792.

(2) Rodrigues, T.; Reker, D.; Schneider, P.; Schneider, G. Counting on natural products for drug design. Nat. Chem. 2016, 8, 531-541.

(3) Patridge, E.; Gareiss, P.; Kinch, M. S.; Hoyer, D. An analysis of FDA-approved drugs: natural products and their derivatives. Drug Discovery Today 2016, 21, 204-207.

(4) Newman, D. J.; Cragg, G. M. Natural products as sources of new drugs over the 30 years from 1981 to 2010. J. Nat. Prod. 2012, 75, $311-335$.

(5) Kapoor, S.; Waldmann, H.; Ziegler, S. Novel approaches to map small molecule-target interactions. Bioorg. Med. Chem. 2016, 24, $3232-3245$.

(6) Schenone, M.; Dancik, V.; Wagner, B. K.; Clemons, P. A. Target identification and mechanism of action in chemical biology and drug discovery. Nat. Chem. Biol. 2013, 9, 232-240.

(7) Reker, D.; et al. Revealing the macromolecular targets of complex natural products. Nat. Chem. 2014, 6, 1072-1078.

(8) Rodrigues, T.; et al. Unveiling (-)-Englerin A as a Modulator of L-Type Calcium Channels. Angew. Chem., Int. Ed. 2016, 55, 1107711081.

(9) Laraia, L.; Robke, L.; Waldmann, H. Bioactive compound collections: from design to target identification. Chem. 2018, 4, 705730.

(10) Laraia, L.; Waldmann, H. Natural product inspired compound collections: evolutionary principle, chemical synthesis, phenotypic screening, and target identification. Drug Discovery Today: Technol. 2017, 23, 75-82.

(11) Reker, D.; Rodrigues, T.; Schneider, P.; Schneider, G. Identifying the macromolecular targets of de novo-designed chemical entities through self-organizing map consensus. Proc. Natl. Acad. Sci. U. S. A. 2014, 111, 4067-4072.

(12) Schneider, G.; et al. Deorphaning the Macromolecular Targets of the Natural Anticancer Compound Doliculide. Angew. Chem., Int. Ed. 2016, 55, 12408-12411.

(13) Rodrigues, T.; Reker, D.; Kunze, J.; Schneider, P.; Schneider, G. Revealing the Macromolecular Targets of Fragment-Like Natural Products. Angew. Chem., Int. Ed. 2015, 54, 10516-10520.

(14) Rodrigues, T.; et al. Machine intelligence decrypts $\beta$-lapachone as an allosteric 5-lipoxygenase inhibitor. Chem. Sci. 2018, 9, 68996903.

(15) Vamathevan, J.; et al. Applications of machine learning in drug discovery and development. Nat. Rev. Drug Discovery 2019, 18, 463477.

(16) Bharadwaj, U.; et al. Drug-repositioning screening identified piperlongumine as a direct STAT3 inhibitor with potent activity against breast cancer. Oncogene 2015, 34, 1341-1353.

(17) Roh, J. L.; et al. Piperlongumine selectively kills cancer cells and increases cisplatin antitumor activity in head and neck cancer. Oncotarget 2014, 5, 9227-9238.

(18) Adams, D. J.; et al. Synthesis, cellular evaluation, and mechanism of action of piperlongumine analogs. Proc. Natl. Acad. Sci. U. S. A. 2012, 109, 15115-15120.

(19) Rodrigues, T.; Sieglitz, F.; Bernardes, G. J. Natural product modulators of transient receptor potential (TRP) channels as potential anti-cancer agents. Chem. Soc. Rev. 2016, 45, 6130-6137.

(20) Akbulut, Y.; et al. (-)-Englerin A is a potent and selective activator of TRPC4 and TRPC5 calcium channels. Angew. Chem., Int. Ed. 2015, 54, 3787-3791.

(21) Li, L.; Zhao, Y.; Cao, R.; Li, L.; Cai, G.; Li, J.; Qi, X.; Chen, S.; Zhang, Z. Activity-based protein profiling reveals GSTO1 as the covalent target of piperlongumine and a promising target for combination therapy for cancer. Chem. Commun. 2019, 55 (30), 4407-4410.

(22) Witte, D. G.; Cassar, S. C.; Masters, J. N.; Esbenshade, T.; Hancock, A. A. Use of a fluorescent imaging plate reader-based calcium assay to assess pharmacological differences between the human and rat vanilloid receptor. J. Biomol. Screening 2002, 7, 466475 .

(23) Reker, D.; Bernardes, G. J. L.; Rodrigues, T. Computational advances in combating colloidal aggregation in drug discovery. Nat. Chem. 2019, 11, 402-418.

(24) Zubcevic, L.; Le, S.; Yang, H.; Lee, S.-Y. Conformational plasticity in the selectivity filter of the TRPV2 ion channel. Nat. Struct. Mol. Biol. 2018, 25, 405-415.

(25) Pumroy, R. A. Molecular mechanism of TRPV2 channel modulation by cannabidiol. bioRxiv, 2019, 521880. https://doi.org/ $10.1101 / 521880$.

(26) Huynh, K. W.; et al. Structure of the full-length TRPV2 channel by cryo-EM. Nat. Commun. 2016, 7, 11130.

(27) Zubcevic, L.; et al. Cryo-electron microscopy structure of the TRPV2 ion channel. Nat. Struct. Mol. Biol. 2016, 23, 180.

(28) Dosey, T. L.; et al. Structures of TRPV2 in distinct conformations provide insight into role of the pore turret. Nat. Struct. Mol. Biol. 2019, 26, 40-49.

(29) Shoemaker, R. H. The NCI60 human tumour cell line anticancer drug screen. Nat. Rev. Cancer 2006, 6, 813-823.

(30) Rees, M. G.; et al. Correlating chemical sensitivity and basal gene expression reveals mechanism of action. Nat. Chem. Biol. 2016, 12, 109-116.

(31) Meyers, R. M.; et al. Computational correction of copy number effect improves specificity of CRISPR-Cas9 essentiality screens in cancer cells. Nat. Genet. 2017, 49, 1779-1784.

(32) N. Cancer Genome Atlas Research. Comprehensive, Integrative Genomic Analysis of Diffuse Lower-Grade Gliomas. N. Engl. J. Med. 2015, 372, 2481-2498.

(33) N. Cancer Genome Atlas Research. Comprehensive genomic characterization defines human glioblastoma genes and core pathways. Nature 2008, 455, 1061-1068. 
(34) Darmanis, S.; et al. Single-Cell RNA-Seq Analysis of Infiltrating Neoplastic Cells at the Migrating Front of Human Glioblastoma. Cell Rep. 2017, 21, 1399-1410.

(35) Zhang, Y.; et al. Purification and Characterization of Progenitor and Mature Human Astrocytes Reveals Transcriptional and Functional Differences with Mouse. Neuron 2016, 89, 37-53.

(36) Oulidi, A.; et al. TRPV2 mediates adrenomedullin stimulation of prostate and urothelial cancer cell adhesion, migration and invasion. PLoS One 2013, 8, No. e64885.

(37) Doñate-Macián, P.; Gómez, A.; Dégano, I. R.; PerálvarezMarín, A. A TRPV2 interactome-based signature for prognosis in glioblastoma patients. Oncotarget 2018, 9, 18400-18409.

(38) Gilbert, L. A.; et al. CRISPR-mediated modular RNA-guided regulation of transcription in eukaryotes. Cell 2013, 154, 442-451.

(39) Liu, J. M.; et al. Piperlongumine selectively kills glioblastoma multiforme cells via reactive oxygen species accumulation dependent JNK and p38 activation. Biochem. Biophys. Res. Commun. 2013, 437, 87-93.

(40) Topf, U.; et al. Quantitative proteomics identifies redox switches for global translation modulation by mitochondrially produced reactive oxygen species. Nat. Commun. 2018, 9, 324.

(41) Conde, J.; Oliva, N.; Artzi, N. Implantable hydrogel embedded dark-gold nanoswitch as a theranostic probe to sense and overcome cancer multidrug resistance. Proc. Natl. Acad. Sci. U. S. A. 2015, 112, E1278-1287.

(42) Conde, J.; Oliva, N.; Atilano, M.; Song, H. S.; Artzi, N. Selfassembled RNA-triple-helix hydrogel scaffold for microRNA modulation in the tumour microenvironment. Nat. Mater. 2016, 15, 353363.

(43) Gilam, A.; et al. Local microRNA delivery targets Palladin and prevents metastatic breast cancer. Nat. Commun. 2016, 7, 12868.

(44) Conde, J.; Oliva, N.; Zhang, Y.; Artzi, N. Local triplecombination therapy results in tumour regression and prevents recurrence in a colon cancer model. Nat. Mater. 2016, 15, 11281138.

(45) Krakstad, C.; Chekenya, M. Survival signalling and apoptosis resistance in glioblastomas: opportunities for targeted therapeutics. Mol. Cancer 2010, 9, 135.

(46) Jacobs, V. L.; Valdes, P. A.; Hickey, W. F.; De Leo, J. A. Current review of in vivo GBM rodent models: emphasis on the CNS1 tumour model. ASN Neuro 2011, 3, No. e00063.

(47) de Vries, N. A.; Beijnen, J. H.; van Tellingen, O. High-grade glioma mouse models and their applicability for preclinical testing. Cancer Treat. Rev. 2009, 35, 714-723.

(48) Snijder, B.; et al. Image-based ex-vivo drug screening for patients with aggressive haematological malignancies: interim results from a single-arm, open-label, pilot study. Lancet Haematol. 2017, 4, e595-e606.

(49) Vladimer, G. I.; et al. Global survey of the immunomodulatory potential of common drugs. Nat. Chem. Biol. 2017, 13, 681-690.

(50) Peralvarez-Marin, A.; Donate-Macian, P.; Gaudet, R. What do we know about the transient receptor potential vanilloid 2 (TRPV2) ion channel? FEBS J. 2013, 280, 5471-5487.

(51) Iwata, Y.; Katayama, Y.; Okuno, Y.; Wakabayashi, S. Novel inhibitor candidates of TRPV2 prevent damage of dystrophic myocytes and ameliorate against dilated cardiomyopathy in a hamster model. Oncotarget 2018, 9, 14042-14057.

(52) Hofmann, L.; et al. The S4-S5 linker - gearbox of TRP channel gating. Cell Calcium 2017, 67, 156-165. 